\title{
OPTIMAL INVESTMENT WITH COSTLY REVERSIBILITY
}

\author{
Andrew B. Abel \\ Janice C. Eberly \\ Working Paper No. 5091
NATIONAL BUREAU OF ECONOMIC RESEARCH
1050 Massachusetts Avenue
Cambridge, MA 02138
April 1995

The authors thank Francisco Delgado for discussions, and seminar participants at Columbia University, the University of Maryland, the University of Montreal, and the Penn Macro Lunch Group for comments. Financial support from the National Science Foundation is gratefully acknowledged. This paper is part of NBER's research program in Economic Fluctuations. Any opinions expressed are those of the authors and not those of the National Bureau of Economic Research.

(C) 1995 by Andrew B. Abel and Janice C. Eberly. All rights reserved. Short sections of text, not to exceed two paragraphs, may be quoted without explicit permission provided that full credit, including $(\mathcal{O}$ notice, is given to the source. 


\title{
OPTIMAL INVESTMENT WITH \\ COSTLY REVERSIBILITY
}

\begin{abstract}
Investment is characterized by costly reversibility when a firm can purchase capital at a given price and sell capital at a lower price. We derive an explicit analytic solution for optimal investment by a firm facing costly reversibility. In addition, we derive a local approximation to the solution which highlights the effects of the parameters of the problem on the triggers for investment. More generally, we extend the Jorgensonian concept of the user cost of capital to the case of uncertainty and define $c_{U}$ and $c_{L}$ as the user costs of capital associated with the purchase and sale of capital, respectively. Optimality requires the firm to purchase and sell capital as needed to keep the marginal revenue product of capital in the closed interval $\left[c_{U}, c_{L}\right]$. This prescription encompasses the case of irreversible investment as well as the standard neoclassical case of costlessly reversible investment. Finally, quantitative analysis suggests that even when the difference between the purchase and sale prices of capital is small, the user costs associated with purchasing and selling capital are closer to those applicable under complete irreversibility than to those applicable under costless reversibility.
\end{abstract}

Andrew B. Abel

Department of Finance

The Wharton School

2315 Steinberg-Dietrich Hall

3620 Locust Walk

University of Pennsylvania

Philadelphia, PA 19104-6367

and NBER
Janice C. Eberly

Department of Finance

The Wharton School

2315 Steinberg-Dietrich Hall

3620 Locust Walk

University of Pennsylvania

Philadelphia, PA 19104-6367

and NBER 


\section{Optimal Investment with Costly Reversibility}

In traditional capital theory, investment is costlessly reversible and the optimal investment policy of a firm maintains the marginal revenue product of capital equal to the Jorgensonian user cost of capital. The firm prevents the marginal revenue product of capital from rising above its user cost by purchasing capital, and it prevents the marginal revenue product of capital from falling below the user cost by selling capital. Recent literature has shown that if investment is completely irreversible, the nature of the optimal investment policy is different: optimal investment can be characterized as a trigger policy, in which capital is purchased to prevent the marginal revenue product of capital from rising above an optimally derived trigger. Values of the marginal revenue product of capital that are lower than the trigger value constitute a range of inaction in which the optimal rate of investment is zero. The boundary of this range of inaction, which is the trigger value for the marginal revenue product of capital, is higher than the Jorgensonian user cost of capital.

Costlessly reversible investment and irreversible investment are opposite ends of a spectrum in which there is costly reversibility. In this paper, we model costly reversibility by introducing a difference between the price at which the firm can purchase capital and the price at which it can sell capital. This wedge between the purchase price and the sale price of capital could arise from transactions costs or from the firm-specific nature of capital. ${ }^{1}$ When the wedge is zero, we have the traditional case of costlessly reversible investment, and when the sale price of capital is zero (so that the wedge is $100 \%$ of the purchase price of capital) we have the case of irreversible investment. Rather than limit attention

${ }^{1}$ A wedge between the purchase and sale prices of capital would also arise if there were adverse selection in the market for used capital goods. However, in this case there would be heterogeneity across firms in purchase and sale prices of capital. 
to these special cases, we study optimal investment in the more general case in which the wedge can be anywhere from zero to $100 \%$ of the purchase price of capital; our model of costly reversibility encompasses the standard models of costlessly reversible investment and irreversible investment. Although this generalization of existing models is one motivation for our analysis, our primary motivation is to achieve an added degree of realism. In his seminal paper on irreversible investment, Arrow (1968) states:

From a realistic point of view, there will be many situations in which the sale of capital goods cannot be accomplished at the same price as their purchase. There are installation costs, which are added to the purchase price but cannot be recovered on sale; indeed, there may on the contrary be additional costs of detaching and moving machinery. Again sufficiently specialized machinery and plant may have little value to others. So resale prices may be substantially below replacement costs. For simplicity, we will make the extreme assumption that resale of capital goods is impossible, so that gross investment is constrained to be non-negative. (pp. 23)

In this paper we abandon the "simplicity" achieved by the "extreme assumption" of irreversibility, yet we are able to derive a tractable solution to the more realistic case of costly reversibility.

We parametrically specify a continuous-time infinitehorizon investment problem in section I. Optimal behavior is easily characterized in terms of the shadow price of capital which we call $q$. Although the actual prices at which the firm can purchase and sell capital are fixed over time, the shadow price $q$ evolves stochastically over time. In particular, $q$ is a function of the underlying fundamentals and is determined by a differential equation with boundary conditions. We present the general solution for $q$ in section II. In section III, we solve 
the boundary conditions and characterize several features of optimal investment behavior analytically.

Optimal behavior is a two-trigger policy in which the firm purchases capital to prevent the marginal revenue product of capital from rising above the upper trigger value and sells capital to prevent the marginal revenue product of capital from falling below the lower trigger value. Values of the marginal revenue product of capital strictly between the two trigger values define a range of inaction in which it is optimal to neither purchase nor sell capital. While the general form of the solution has been pointed out in a model of investment by Bertola (1988) and in a model of entry and exit by Dixit (1989), our analytic solution focuses attention on the size of the range of inaction, which is measured by the ratio of the upper trigger to the lower trigger. We show in section III that this ratio is a strictly increasing function of the ratio of the purchase price of capital to the sale price of capital. Moreover, the ratio of the upper trigger to the lower trigger is larger than the ratio of the purchase price of capital to the sale price of capital. Indeed, we show that the derivative of the trigger ratio with respect to the capital price ratio is infinite when the capital price ratio is one. Thus, introducing even a tiny wedge between the purchase price and the sale price of capital has a substantial effect on the trigger ratio and introduces a substantial range of inaction.

In section IV we generalize the concept of the user cost of capital introduced in the case of costlessly reversible investment by Jorgenson (1963). When there is a wedge between the purchase and sale prices of capital, there are two notions of the user cost of capital: (1) a user cost, $c_{U}$, which is relevant for purchasing capital; and (2) a user cost, $c_{L}$, which is relevant for selling capital. The concept of user cost allows us to state the optimal two-trigger policy as a simple rule: Purchase capital as needed to prevent the marginal revenue product of capital from rising above the user cost $c_{U}$, sell capital as needed to prevent the marginal revenue product from falling below the user cost $c_{L}$, and neither purchase nor sell capital if the marginal revenue product of capital is strictly between $c_{L}$ and $c_{U}$. In section $\mathrm{V}$ we show that this rule 
encompasses the prescription for optimal investment in the case of costlessly reversible investment discussed by Jorgenson (1963) as well as the prescription for optimal investment in the burgeoning literature on irreversible investment (Bertola (1988), Bertola and Caballero (1994), Pindyck (1988, 1991), Dixit (1989), and Dixit and Pindyck (1994)).

The solution for optimal behavior derived in section III has a simple recursive structure. The first step solves for the ratio of the upper trigger value to the lower trigger value of the marginal revenue product of capital. The individual trigger values are then calculated as closed-form functions of this ratio. Finally, these values are used in the closed-form solution for $q$. Although much of the solution is characterized by closed-form equations, the ratio of the upper trigger value to the lower trigger is determined as the root of a nonlinear equation. Our analysis of irreversibility in section $\mathrm{V}$ leads to the development of a simple algorithm to compute this root.

The analytic solution derived in section III does not offer a transparent view of the effect of various parameters on optimal investment behavior. Section VI makes some progress in this direction by presenting a local approximation to the solution. The ratio of the upper and lower trigger values is shown to be a locally cubic function of the ratio of the purchase and sale prices of capital. The trigger ratio is also shown to be an increasing function of the variance of the shocks facing the firm and a decreasing function of the discount rate and the depreciation rate of capital.

In section VII we explore the quantitative significance of costly reversibility. We find that even relatively small differences between the purchase and sale prices of capital can cause the investment triggers to be closer to the triggers that would hold under complete irreversibility than to the triggers that would hold under costless reversibility. Equivalently, the assumption of complete irreversibility can lead to better a quantitative description of investment behavior than the conventional assumption of costless reversibility, even when the wedge between the purchase and sale prices of capital is modest. 
Concluding remarks are presented in section VIII, and the multi-part Appendix is used to keep lengthy algebraic derivations out of the main text.

\section{The Firm's Optimization Problem}

Consider a firm that produces output at time $t$ using its capital stock $K_{t}$ and variable factors of production. The firm sells all of its output, and the price of its output is determined by a demand curve that depends on the random variable $X_{t}$. Assume that the operating profit of the firm, i.e., revenue minus the cost of the variable factors of production, is given by

$$
\pi\left(K_{i}, X_{i}\right)=\frac{h}{1-\gamma} X_{i}^{\gamma} K_{+}^{1-\gamma} \text { where } h>0 \text { and } 0<\gamma<1 .
$$

The specification in equation (1) can be derived for a firm with a constant-returns-to-scale Cobb-Douglas production function facing an isoelastic demand curve. ${ }^{2}$ It has been used, for example, by Bertola (1988) and Dixit (1991) in their analyses of irreversible investment.

According to equation (1), the instantaneous operating profit of the firm depends on the firm's capital stock $K_{t}$ and on the stochastic component of demand $X_{t}$. Thus, the evolution of the operating profit depends on the evolution of $X_{t}$ and $K_{t}$ over time. The demand shock $X_{t}$ is assumed to evolve exogenously according to a geometric Brownian motion

$$
\frac{d X_{t}}{X_{t}}=\mu_{x} d t+\sigma_{x} d z, \quad \sigma_{x}>0
$$

${ }^{2}$ Suppose that the production function is $Q=L^{\alpha} K^{1-\alpha}$, where $Q$ is output, $L$ is labor, $K$ is capital, and $0<\alpha<1$, and the demand curve is $P=X^{1-v} Q^{v-1}$ where $P$ is the price of output, $X$ is the demand shock, and $0<v<1$. It can be shown that operating profit, $\max _{L}(P Q-w L)$, where $w$ is the wage, is equal to $h X^{\gamma} K^{1-\gamma}$, where $h \equiv(1-\alpha v)\left(\frac{\alpha v}{w}\right)^{\frac{\alpha v}{1-\alpha v}}$ and $\gamma \equiv \frac{1-v}{1-\alpha v}$. 
where $d z$ is an increment to a standard Wiener process, with $E\{d z\}=0$ and $(d z)^{2}=d t$.

We assume that the firm is risk-neutral and maximizes the expected present value of its cash flow over an infinite horizon. Its cash flow at any point in time equals the operating profit, $\pi\left(K_{t}, X_{t}\right)$, minus the cost of purchasing capital at a constant price $b_{U}>0$, plus the proceeds received from selling capital at a constant price $b_{L} \geq 0$. In order for the firm's optimization problem to be well-posed, we assume that $b_{L} \leq$ $b_{U}{ }^{3}$ Because the cost of adjustment is (piecewise) linear, the rate of investment or disinvestment can be infinite. ${ }^{4}$ The evolution of the capital stock $K_{t}$ is therefore given by

$$
d K_{t}=I_{t}-\left(\delta K_{t}\right) d t
$$

where $l_{t}$ is gross investment at time $t$. More precisely, let $U_{t}$ be a nondecreasing function of time representing the cumulation of all positive gross investment up to time $t$, and let $L_{t}$ be a nonincreasing function of time representing the cumulation of all negative gross investment up to time $t$. Defining $I_{t}^{+} \equiv d U_{t}$ and $I_{t}^{-} \equiv d L_{t}$, gross investment is $I_{t} \equiv I_{t}^{+}+I_{t}^{-}$. The capital stock depreciates at a constant proportional rate $\delta \geq 0$ so that $\left(\delta K_{t}\right) d t$ is the amount of depreciation that occurs over a small interval of time $d t$.

Assuming that the firm discounts future cash flows at the constant rate $r>0$, and that $r>\mu_{X},{ }^{5}$ the value of the firm is ${ }^{6}$

${ }^{3}$ If this assumption were violated so that $b_{L}>b_{U}$, the firm could earn infinite profit by purchasing capital at price $b_{U}$ and then selling it immediately at the higher price $b_{L}$.

${ }^{4}$ The capital stock therefore follows a continuous, but non-differentiable, path.

${ }^{5}$ Under optimal policy the marginal revenue product of capital, $h X^{\gamma} K^{-\gamma}$, satisfies $c_{L} \leq h X^{\gamma} K^{-\gamma} \leq c_{U}$ where $c_{L}$ and $c_{U}$ are constants given in equations (28) and (25) respectively. Recalling that $0<\gamma<1$, so that $(\gamma-1) / \gamma<0$, this inequality is equivalent to $c_{L}^{(\gamma-1) / \gamma} \geq h^{(\gamma-1) / \gamma} X^{\gamma-1} K^{1-\gamma} \geq c_{U}^{(\gamma-1) / \gamma}$.

Multiplying this inequality by $\left(h^{1 / \gamma /(1-\gamma)) X}\right.$ and recalling that $\pi(K, X)=(h /(1-\gamma)) X K^{1-\gamma}$, we obtain 
$V\left(K_{t}, X_{,}\right) \equiv \max _{\left\{r_{t+s}, I_{t+s}\right\}} E_{t}\left\{\int_{0}^{-} e^{-r s}\left[\pi\left(K_{t+s}, X_{t+s}\right) d s-b_{U} I_{t+s}^{+}-b_{L} I_{t+1}^{-}\right]\right\}$.

Since $U_{t}$ and $L_{t}$ are not differentiable, the last two terms in equation (3) are to be interpreted as Stieltjes integrals.

The Bellman equation associated with the optimization problem in equation (3) is

$$
r V(K, X)=\frac{h}{1-\gamma} X^{\gamma} K^{2-\gamma}-\delta K V_{x}(K, X)+\mu_{x} X V_{x}(K, X)+\frac{1}{2} \sigma_{x}^{2} X^{2} V_{x x}(K, X)^{\circ}
$$

The left hand side of equation (4) is the required return on the firm. The right hand side of this equation is the expected actual return consisting of the cash flow, $[h /(1-\gamma)] X^{\gamma_{K}}{ }^{1-\gamma}$, plus the expected change in the value of the firm, $-\delta K V_{K}(K, X)+$ $\mu_{X} X V_{X}(K, X)+(1 / 2) \sigma^{2} X^{2} V_{X X}(K, X){ }^{7}$

$\left(h^{1 / \gamma} /(1-\gamma)\right) c_{L}{ }^{(\gamma-1) / \gamma} X \geq \pi(X, K) \geq\left(h^{1 / \gamma} /(1-\gamma)\right) c_{U}{ }^{(\gamma-1) / \gamma} X$.

Since the expected growth rate of $X$ is $\mu_{X}$, the operating profit of the firm is bounded above and bounded below by processes with expected growth rates equal to $\mu_{x}$. Therefore, $\lim _{s \rightarrow \infty} e^{-n} E_{t}\left\{\pi\left(K_{t+s}, X_{t+s}\right)\right\}=0$ if and only if $\mu_{X}<r$. Thus the expected present value of operating profits, $E_{i}\left\{\int_{0}^{\infty} e^{-r s} \pi\left(K_{t+s}, X_{t+s}\right) d s\right\}$, is finite if and only if $\mu_{X}<r$.

${ }^{6}$ Suppose that the firm begins at time 0 with an initial capital stock $K_{0}$. If the marginal valuation of capital $V_{K}\left(K_{0}, X_{0}\right)$ is less than $b_{L}$, the firm immediately sells enough capital to make the marginal valuation equal to $b_{L}$. If $V_{K}\left(K_{0}, X_{0}\right)>b_{U}$, the firm immediately buys enough capital to make the marginal valuation of capital equal to $b_{U}$. If $b_{L} \leq V_{K}\left(K_{0}, X_{0}\right) \leq b_{U}$, there is no jump in the capital stock at time 0.

${ }^{7}$ The right hand side of equation (4) does not contain any terms reflecting the cash flows associated with purchasing or selling capital. Each unit of capital purchased by the firm increases the value of the firm by $V_{K}(K, X)$ and costs $b_{U}$, so the contribution of positive investment to the maximand in equation (3) is $I^{+}\left(V_{K}(K, X)-b_{U}\right)$. As shown later, under the 
Equation (4) holds identically in $K$. Thus, the partial derivative of the left hand side with respect to $K$ equals the partial derivative of the right hand side with respect to $K$. Performing this partial differentiation yields

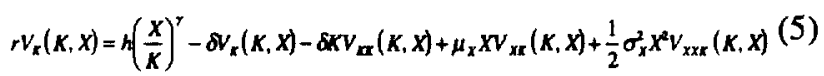

Equation (5) characterizes the marginal valuation of capital $V_{K}(K, X)$. To get a simpler expression for the marginal valuation of capital, define $y \equiv X / K$, and note that the marginal revenue product of capital is $h y \gamma$. When the marginal valuation of capital is in the interior of the interval $\left[b_{L}, b_{U}\right]$, gross investment is zero, and the evolution of $y$ is given by

$$
\frac{d y}{y}=\mu_{y} d t+\sigma_{y} d z \text { where } \mu_{y} \equiv \mu_{x}+\delta \text { and } \sigma_{y} \equiv \sigma_{x} \text {. }
$$

When the marginal valuation of capital reaches a boundary of the interval $\left[b_{L}, b_{U}\right]$, so that gross investment is non-zero, investment occurs to prevent the marginal valuation of capital from leaving this interval. The precise form of this policy will be analyzed below with the boundary conditions for the investment problem.

In our parametric specification of the optimization problem, the value function $V(K, X)$ is homogeneous of degree one in $K$ and $X$. Thus the marginal valuation of capital, $V_{K}(K$, $X$ ), is homogenous of degree zero in $K$ and $X$, and hence can

optimal policy $I^{+}$is nonzero only when the marginal valuation of capital $V_{K}(K, X)$ equals the purchase price of capital $b_{U}$ so that $I^{+}\left(V_{K}(K, X)-b_{U}\right)$ is always zero. Similarly, each unit of capital sold by the firm increases the value of the firm by $b_{L}$ $V_{K}(K, X)$, so the contribution of negative investment to the maximand in equation (3) is $-I^{-}\left(b_{L}-V_{K}(K, X)\right)$. As shown later, under the optimal policy $I^{-}$is nonzero only when the marginal valuation of capital $V_{K}(K, X)$ equals the sale price of capital $b_{L}$ so that $-I^{-}\left(b_{L}-V_{K}(K, X)\right)$ is always zero. Thus, the Bellman equation in (4) holds for nonzero as well as zero optimal investment. 
be written simply as a function of $y$, the ratio of $X$ to $K$. Define $q(y)$ as the marginal valuation of capital

$$
q(y) \equiv V_{K}(K, X) \text {. }
$$

Differentiating the definition in equation (7) and using the definition of $y$ yields expressions for the following higherorder partial derivatives of the value function

$$
\begin{aligned}
& V_{K K}(K, X)=\frac{-y q^{\prime}(y)}{K} \\
& V_{x K}(K, X)=\frac{q^{\prime}(y)}{K} \\
& V_{x X K}(K, X)=\frac{q^{\prime \prime}(y)}{K^{2}} .
\end{aligned}
$$

Substituting the definition of $y$, and equations $(7,8 a, 8 b$, $8 c$ ) into equation (5) yields a second-order ordinary differential equation for the marginal valuation of capital $q(y)$,

$$
(r+\delta) q(y)=h y^{\gamma}+\mu_{y} y q^{\prime}(y)+\frac{1}{2} \sigma_{y}^{2} y^{2} q^{\prime \prime}(y)
$$

In addition to satisfying the differential equation (9), $q(y)$ must satisfy the boundary conditions. ${ }^{8}$ Recall that optimal investment is zero when the marginal valuation of capital $q(y)$ is in the interior of the interval $\left[b_{L}, b_{U}\right]$. The firm will undertake nonzero gross investment only if $q(y)$ reaches one of the boundaries $b_{L}$ or $b_{U}$. The values of $y$ at these boundaries, $y_{L}$ and $y_{U}$, are given by the smooth-pasting conditions

$$
\begin{aligned}
& q\left(y_{L}\right)=b_{L} \\
& q\left(y_{u}\right)=b_{U} .
\end{aligned}
$$

In addition to satisfying the smooth-pasting conditions, $q(y), y_{L}$, and $y_{U}$ must satisfy the high-contact conditions

${ }^{8}$ See Dumas (1991) for a clear presentation of the smooth-pasting and high-contact conditions used below. 


$$
\begin{aligned}
& q^{\prime}\left(y_{L}\right)=0 \\
& q^{\prime}\left(y_{u}\right)=0 .
\end{aligned}
$$

The high-contact conditions guarantee that if the upper boundary is reached at time $t$, the marginal valuation of an additional unit of capital, $q$, will equal its cost, $b_{U}$, at both $K_{t}$ and $K_{t+}$. Similarly, if the lower boundary is reached at time $t$, $q$ must equal the resale price of capital, $b_{L}$, both at $K_{t}$ and $K_{t+}$. The high-contact conditions therefore ensure that the marginal valuation of an additional unit of capital, $q$, does not change when investment is nonzero.

\section{The General Solution for $q(y)$}

Our strategy in solving for the marginal valuation of capital, $q(y)$, is to obtain the general solution to the differential equation (9) and then to use the four boundary conditions in equations $(10 a, b)$ and $(11 a, b)$ to determine the two free parameters in the general solution and to determine the values of the triggers $y_{L}$ and $y_{U}$.

The general solution to the second-order ordinary differential equation (9) involves the roots of the following quadratic equation (see, for example, Dixit and Pindyck (1994))

$$
\rho(\eta) \equiv-\frac{1}{2} \sigma_{y}^{2} \eta^{2}-\left(\mu_{y}-\frac{1}{2} \sigma_{y}^{2}\right) \eta+r+\delta=0
$$

Note that $\rho(\eta)$ is strictly concave, $\rho(0)=r+\delta>0$, and $\rho(1)$ $=r+\delta-\mu_{y}>0$. Thus, $\rho(\gamma)>0$. Also, note that $\rho(\eta)=0$ has two distinct roots, $\alpha_{P}>0$ and $\alpha_{N}<0$, which satisfy ${ }^{9}$

$$
\alpha_{N}<0<\gamma<1<\alpha_{P} \text {. }
$$

Using the roots of the quadratic equation $\rho(\eta)=0$, we can express the general solution to the differential equation (9) as

${ }^{9}$ Other properties of the roots of equation (12) are given in Part A of the Appendix. 


$$
q(y)=H y^{\gamma}+\hat{A} y^{\alpha_{\mu}}+\hat{B} y^{\alpha_{r}}, \text { where } H \equiv \frac{h}{\rho(\gamma)}>0
$$

and $\hat{A}$ and $\hat{B}$ are constants that will be determined by the boundary conditions. It is straightforward to verify that the expression for $q(y)$ in equation (14) solves the differential equation (9).

Recall that $q(y)$ is defined as $V_{K}(K, X)$, the marginal valuation of a unit of capital. The marginal valuation of capital equals the expected present value of marginal revenue products of capital under optimal behavior, and equation (14) expresses this expected present value as the sum of three components: (1) The term $H_{y}{ }^{\gamma}$ is the expected present value of marginal revenue products of capital if the firm were prevented from ever purchasing or selling capital; (2) If the firm has the option to purchase but not sell capital, this opportunity to increase the capital stock in the future decreases expected future marginal revenue products and changes the expected present value of marginal revenue products by $\hat{B} y^{\alpha_{P}}$, where the constant $\hat{B}$ is negative (see equation (20b) below); and (3) If the firm has the option to sell but not purchase capital, this opportunity to reduce the future capital stock increases expected future marginal revenue products of capital and increases the expected present value of future marginal revenue products by $\hat{A} y^{\alpha_{N}}$, where the constant $\hat{A}$ is positive (see equation (20a) below). In the general case of costly reversibility the firm can both purchase and sell capital, so the marginal value of capital equals $H_{y}^{\gamma}$ plus the effects of the call option to purchase capital $\left(\hat{B} y^{\alpha_{P}}\right)$ and the put option to sell capital $\left(\hat{A} y^{\alpha_{N}}\right.$ ). Bertola (1988) uses contingent claims pricing to derive the general forms of these option terms but does not derive explicit expressions for the constants $\hat{A}$ and $\hat{B}$. In the next section, we use the boundary conditions to obtain explicit expressions for these constants. 


\section{Solving the Boundary Conditions}

The four boundary conditions $(10 a, b)$ and $(11 a, b)$ determine the constants $\hat{A}$ and $\hat{B}$ in equation (14) as well as the values of $y_{L}$ and $y_{U}$. These equations have a recursive structure that we exploit by defining $G \equiv \frac{y_{U}}{y_{L}}$ as the ratio of the $y_{2}$

upper boundary on $y$ to the lower boundary on $y$. Recall that the marginal revenue product of capital is $h y{ }^{\gamma}$ so that $h y_{L}^{\gamma}$ is the lower trigger and $h y_{U}^{\gamma}$ is the upper trigger on the marginal revenue product of capital. Therefore, $G^{\gamma}$ is the ratio of the upper and lower triggers on the marginal revenue product of capital.

The value of $G$ (and hence of $G^{\gamma}$ ) is given by the root of an equation that does not depend on $\hat{A}, \hat{B}, y_{L}$, or $y_{U}$. After we obtain the value of $G$, then we compute $y_{L}$ and $y_{U}$ using closed-form functions of $G$. Finally, we compute $\hat{A}$ and $\hat{B}$ using closed-form functions of $G, y_{L}$, and $y_{U}$. Part F of the Appendix verifies that the solution presented in this section actually solves the differential equation ( 9 ) and the boundary conditions $(10 a, b)$ and $(11 a, b)$.

\section{III.A. The Solution for $G$}

In order to simplify the notation in the subsequent analysis we define the function $\theta(x)$ for $x \geq 0$ as

$$
\theta(x) \equiv \frac{x^{\alpha_{r}}-x^{\gamma}}{x^{\alpha_{p}}-x^{\alpha_{N}}} .
$$

In light of equation (13) it is obvious that $0 \leq \theta(x) \leq 1$. It is straightforward to show that

$$
\theta(0)=0<\theta(1)=\frac{\alpha_{P}-\gamma}{\alpha_{P}-\alpha_{N}}<1=\theta(\infty)
$$


In Part B of the Appendix ${ }^{10}$ we show that $\theta(x)$ is a monotonically increasing function of $x$.

The optimal value of the ratio $G$ depends on $R \equiv \frac{b_{U}}{b_{l}}$ which is the ratio of the purchase price of capital to the sale price of capital faced by the firm. Given $R \geq 1$, the value of $G$ is defined implicitly by ${ }^{11}$

$$
J(R, G) \equiv R-G^{\gamma}-\frac{\gamma}{\alpha_{N}}\left(R-G^{\alpha_{\mu}}\right) \theta(G)-\frac{\gamma}{\alpha_{P}}\left(R-G^{\alpha_{r}}\right)(1-\theta(G))=0 .(17)
$$

Proposition 1. For any $R \geq 1$, there exists a unique ${ }^{12} G \geq 1$, denoted $G(R)$, such that $J(R, G)=0 . G(1)=1$, and the function $G(R)$ is strictly increasing for $R>1$.

Proof. See Part G of the Appendix.

Proposition 1 states that the wedge (expressed as a ratio) between the upper trigger $y_{U}$ and the lower trigger $y_{L}$ is a strictly increasing function of the wedge (expressed as a ratio) between the purchase price of capital $b_{U}$ and the sale price of capital $b_{L}$. Proposition 2, which follows, implies that even a tiny wedge between the purchase and sale prices of capital can introduce a substantial wedge between the upper and lower triggers.

Proposition 2. $G^{\prime}(1)=\infty$.

${ }^{10}$ See especially equations (B.9) and (B.12).

${ }^{11}$ In Part $F$ of the Appendix we verify that equation (17) characterizes the optimal value of $G$.

${ }^{12}$ In discussing the values of the two triggers, Bertola (1988) remarks, "Although it does not seem possible to prove analytically the uniqueness of their solution, numerical procedures are not at all sensitive to the starting point of the iteration, suggesting that the solution is indeed unique." Our Proposition 1 proves the uniqueness of $G$, the ratio of the triggers, and later we show that the triggers are closed-form functions of $G$, which implies that the triggers are unique, as conjectured by Bertola. 
Proof. Assume that $R=1$. Therefore $G=1$. In Part $\mathrm{D}$ of the Appendix it is shown that $\left.J_{G}(1,1)\right|_{J=0}=0$ (see equation D.16) and that $J_{R}(1,1)>0$ (see equation D.8). Therefore, the application of the implicit function theorem (equation G.1 in the Appendix) shows that $G^{\prime}(1)=\infty$. q.e.d.

Interpreting $R-1$ as a transaction cost, Proposition 2 states that the derivative of the (geometric) distance between the upper and lower triggers with respect to the transaction cost is infinite when evaluated at zero transaction cost. A similar finding is also reported by Dixit $(1989$, p. 630$)$ in a model of entry and exit.

III.B. The Triggers $y_{U}$ and $y_{L}$

Now that we have solved for $G$, the ratio of the triggers, we can compute their individual values. It is most convenient to express the triggers in terms of triggers on the marginal revenue product of capital $h y^{\gamma}$. The firm pursues an investment policy that prevents the marginal revenue product of capital from falling below the lower trigger $h y_{L}^{\gamma}$ and from rising above the upper trigger $h y_{u}^{\gamma}$. The presentation of the triggers is simplified by defining the function $\Psi(x)$

$$
\Psi(x) \equiv(r+\delta) \frac{\alpha_{p}-\gamma}{\alpha_{p}-\gamma \frac{(1-\theta(x))}{(1-\theta(1))}}>0
$$

Recall that $r+\delta>0, \alpha_{P}>\gamma>0,1-\theta(1)>0$, and $\theta^{\prime}(x)>0$ so that inspection of equation (18) reveals that $\Psi(x)$ is a strictly decreasing function of $x$.

As verified in Part $F$ of the Appendix, the trigger values for the marginal revenue product of capital are

$$
\begin{aligned}
& h y_{L}^{\gamma}=\Psi(G) b_{L} \\
& h y_{U}^{\gamma}=\Psi\left(G^{-1}\right) b_{U}
\end{aligned}
$$


Observe that $G^{\gamma}$ is the ratio of the trigger values of the marginal revenue product of capital. The following proposition shows that if $R$, the ratio of the purchase and sale prices of capital faced by the firm, is greater than one, then $G^{\gamma}$ is larger than $R$.

Proposition 3. $\frac{G^{\gamma}}{R}=\frac{\Psi\left(G^{-1}\right)}{\Psi(G)} \geq 1$, with strict inequality if $R>1$.

Proof. Divide each side of equation (19b) by the corresponding side of equation (19a) to obtain $G^{\gamma}=\frac{h y_{U}^{\gamma}}{h y_{L}^{\gamma}}=\frac{\Psi\left(G^{-1}\right) b_{U}}{\Psi(G) b_{L}}=\frac{\Psi\left(G^{-1}\right)}{\Psi(G)} R$. If $R>1$, then $G>1$

(Proposition 1). Therefore, $G>G^{-1}$, and $\Psi\left(G^{-1}\right)>\Psi(G)$ because $\Psi(x)$ is strictly decreasing in $x$. q.e.d.

\section{III.C. The Constants $\hat{A}$ and $\hat{B}$}

We can now complete our solution by computing $\hat{A}$ and $\hat{B}$ using closed-form functions of $G, y_{L}$, and $y_{U}$. Specifically $\hat{A}$ and $\hat{B}$ are given by

$$
\hat{A}=A \theta(G) y_{L}^{\gamma-\alpha_{N}}=A \theta\left(G^{-1}\right) y_{U}^{\gamma-\alpha_{N}},
$$

where $A \equiv-\frac{\gamma H}{\alpha_{N}}>0$, and

$\hat{B}=B(1-\theta(G)) y_{L}^{\gamma-\alpha_{r}}=B\left(1-\theta\left(G^{-1}\right)\right) y_{U}^{\gamma-\alpha_{P}}$,

where $B \equiv-\frac{\gamma H}{\alpha_{P}}<0$.

Notice that equation (20a) presents two alternative equivalent expressions for $\hat{A}$, and equation (20b) presents two alternative equivalent expressions for $\hat{B} \cdot{ }^{13}$ Although either of

${ }^{13}$ The two expressions for $\hat{A}$ are equivalent because $A \theta(G) y_{L}^{\gamma-\alpha_{N}}=A \theta(G) G^{\alpha_{N}-\gamma} y_{U}^{\gamma-\alpha_{N}}=A \theta\left(G^{-1}\right) y_{U}^{\gamma-\alpha_{N}}$ where the

first equality follows from $y_{U}=G y_{L}$, and the second equality 
the equivalent expressions will serve our purposes in most cases, in special cases examined in section V, one or the other of the equivalent alternatives turns out to be particularly useful. Substituting the expressions for $\hat{A}$ and $\hat{B}$ into equation (14) yields the following equivalent expressions for $q(y)$

$$
\begin{aligned}
& q(y)=H y^{\gamma}+A \theta(G) y_{L}^{\gamma}\left(\frac{y}{y_{L}}\right)^{\alpha_{N}}+B(1-\theta(G)) y_{L}^{\gamma}\left(\frac{y}{y_{L}}\right)^{\alpha_{r}} \\
& q(y)=H y^{\gamma}+A \theta\left(G^{-1}\right) y_{U}^{\gamma}\left(\frac{y}{y_{U}}\right)^{a_{H}}+B\left(1-\theta\left(G^{-1}\right)\right) y_{U}^{\gamma}\left(\frac{y}{y_{U}}\right)^{\alpha_{P}} .
\end{aligned}
$$

\section{The User Cost of Capital}

In the standard neoclassical model of costlessly reversible investment, the optimal policy is to adjust the capital stock continuously to maintain the marginal revenue product of capital equal to the user cost of capital. In a deterministic framework, Jorgenson (1963) showed that the user cost of capital is given by

$$
c_{J} \equiv\left(r+\delta-\frac{\dot{p}_{K}}{p_{K}}\right) p_{K},
$$

where $p_{K}$ is the price at which the firm can purchase or sell capital. The user cost has three components: (1) interest cost, $r p_{K}$; (2) physical depreciation, $\delta p_{K}$; and (3) the capital loss associated with the decline in the price of a unit of capital, $-\dot{p}_{K}$. Of course, if $p_{K}$ is constant, the Jorgensonian user cost is simply $(r+\delta) p_{K}$.

follows from equation (E.7) in the Appendix. The two

expressions for $\hat{B}$ are equivalent because

$B(1-\theta(G)) y_{L}^{\gamma-\alpha_{s}}=B(1-\theta(G)) G^{\alpha_{s-\gamma}} y_{u}^{\gamma-\alpha_{s}}=B\left(1-\theta\left(G^{-1}\right)\right)_{y_{u}^{\gamma-\alpha_{s}}}$

where the first equality follows from $y_{U}=G y_{L}$, and the second equality follows from equation (E.8) in the Appendix. 
In this section we extend the Jorgensonian concept of the user cost of capital to the general case of costly reversibility under uncertainty. When $R>1$, so that there is a wedge between the purchase and sale prices of capital, there is a user cost relevant for the purchase of capital and a user cost relevant for the sale of capital.

First consider the user cost relevant for the purchase of capital. As we have shown, the firm purchases capital only when $y=y_{U}$. The user cost of capital relevant for purchasing capital when $y=y_{U}$ is

$$
c_{U} \equiv\left(r+\delta-\frac{E\left\{d q \mid y=y_{U}\right\} / d t}{q\left(y_{U}\right)}\right) b_{U}
$$

The expression for $c_{U}$ in equation (23) is analogous to the Jorgensonian user cost in equation (22). The components of the user cost representing interest, $r b_{U}$, and depreciation, $\delta b_{U}$, are the same as in the Jorgensonian user cost. The third component of the user cost, $\frac{-E\left\{d q \mid y=y_{u}\right\} / d t}{q\left(y_{v}\right)} b_{v}$, measures the expected capital loss on a unit of capital as the expected change in the shadow price of capital, $q$, rather than as the expected change in the actual purchase price. ${ }^{14}$ When the firm is purchasing capital, the expected capital loss (per unit of time) is $-E\left\{d q \mid y=y_{U}\right\} / d t$. Recall from the smooth-pasting condition in equation (10b) that $b_{U}\left(q\left(y_{U}\right)=1\right.$, so that the

${ }^{14}$ In the case of costlessly reversible investment studied by Jorgenson (1963), the shadow price of capital, $q$, is always equal to the purchase and sale price of capital, $p_{K}$, so there is no distinction between shadow price and actual price. However, in the case of costly reversibility, as well as in the case of complete irreversibility, the shadow price and actual price of capital differ unless the firm is actually purchasing or selling capital. When the shadow price differs from the actual price of capital, it is the shadow price of capital that is relevant for the user cost of capital. 
expected capital loss per unit of time is $\frac{-E\left\{d q \mid y=y_{U}\right\} / d t}{q\left(y_{U}\right)} b_{U}$.

Notice that the expected capital loss when $y=y_{U}$ is positive because a decrease in $X$ (recall that $y \equiv X / K$ ) will cause the shadow price of capital, $q(y)$, to fall, but an increase in $X$ will induce the firm to increase its capital stock in order to prevent $q(y)$ from rising above $b_{U}$. Thus, the expected value of the change in $q$, given that $q$ is equal to its upper barrier, is negative, and hence the expected capital loss is positive. This expected capital loss causes the user cost $c_{U}$ to be higher than $(r+\delta) b_{U}$, which is the Jorgensonian user cost in the presence of a constant price of capital, $b_{U}$.

We can compute a closed-form expression for ${ }^{c} U$ using the following expression for the expected change in $q(y)$ when $y$ is at either the upper or lower boundary

$$
E\left\{d q \mid y=y_{i}\right\} / d t=\frac{1}{2} \sigma_{y}^{2} y_{i} q^{\prime \prime}\left(y_{i}\right) \quad \text { for } i=L, U .
$$

As shown in Part $H$ of the Appendix, equation (24) is derived by applying Ito's Lemma to the function $q(y)$ and then using the high-contact conditions (equations $(11 \mathrm{a}, \mathrm{b})$ ) which state that $q^{\prime}\left(y_{i}\right)=0$ for $i=L, U$. It is also shown that applying equation (24) to the expression for $q(y)$ in equation (21b) and substituting the result into the definition of $c_{U}$ yields

$$
c_{u}=\Psi\left(G^{-1}\right) b_{u} .
$$

Recall from equation (19b) that the upper trigger value of the marginal revenue product of capital is $h y_{u}^{y}=\Psi\left(G^{-1}\right) b_{U}$. In light of equation (25) this trigger value can be stated simply as

$$
h y_{U}^{\gamma}=c_{U} \text {. }
$$

We now turn our attention to the user cost relevant for selling capital. We define this user cost as

$$
c_{L} \equiv\left(r+\delta-\frac{E\left\{d q \mid y=y_{L}\right\} / d t}{q\left(y_{L}\right)}\right) b_{L} .
$$


This definition is analogous to the definition of $c_{U}$, except that the sale price of capital $b_{L}$ replaces the purchase price of capital $b_{U}$, and the expected capital loss is computed at $y_{L}$, the lower boundary for $y$. Notice that when $y=y_{L}$, so that $q(y)$ is at its lower boundary $b_{L}$, an increase in $X$ will increase $q(y)$ but a decrease in $X$ will not decrease $q(y)$ because the firm will sell capital to prevent $y$ from falling below $y_{L}$. With the possibility of an increase in $q(y)$, and no possibility of a decrease in $q(y)$, the expected change in $q(y)$ is positive. This expected capital gain makes the user cost of capital $c_{L}$ lower than the corresponding Jorgensonian user $\operatorname{cost}(r+\delta) b_{L}$.

The analogs to equations (25) and (26) are derived in Part $\mathrm{H}$ of the Appendix. The user cost of capital relevant for selling capital is

$$
c_{L}=\Psi(G) b_{L},
$$

and thus the lower trigger value for the marginal revenue product of capital is

$$
h y_{L}^{\gamma}=c_{L} \text {. }
$$

The two user costs, $c_{L}$ and $c_{U}$, allow us to characterize optimal investment behavior by a simple rule: Keep the marginal revenue product of capital from leaving the closed interval $\left[c_{L}, c_{U}\right] \equiv\left[\Psi(G) b_{L}, \Psi\left(G^{-1}\right) b_{U}\right]$. To implement this rule, purchase capital to prevent its marginal revenue product from rising above the user cost $c_{U}$, and sell capital to prevent its marginal revenue product from falling below $c_{L}$. If the marginal revenue product of capital is in the interior of the interval $\left[c_{L}, c_{U}\right]$, then it is optimal to neither purchase nor sell capital.

\section{Polar Cases: Irreversibility and Costless Reversibility}

We have shown that in the general case of costly reversibility, optimal investment behavior is characterized by a two-trigger policy that prevents the marginal revenue product of capital from leaving the interval $\left[\Psi(G) b_{L}, \Psi\left(G^{-1}\right) b_{U}\right]$. In 
this section, we show how this characterization of optimal investment also applies to the special cases of irreversibility and costless reversibility.

We begin our analysis of special cases by using equation (16) to evaluate the function $\Psi(x)$ at $x=0, x=1$, and $x=\infty$

$$
\begin{aligned}
& \Psi(0)=(r+\delta)\left(1-\frac{\gamma}{\alpha_{N}}\right) \\
& \Psi(1)=r+\delta \\
& \Psi(\infty)=(r+\delta)\left(1-\frac{\gamma}{\alpha_{p}}\right)
\end{aligned}
$$

We consider three special cases. First, suppose that investment is costlessly reversible as in standard neoclassical theory. In this case, $b_{U}=b_{L}$ and hence $R=1$. Therefore, it follows from Proposition 1 that $G=1$ which implies that $\Psi(G)$ $=\Psi\left(G^{-1}\right)=\Psi(1)=r+\delta$. Letting $p_{K}$ denote the common value of $b_{U}$ and $b_{L}$, the interval $\left[\Psi(G) b_{L}, \Psi\left(G^{-1}\right) b_{U}\right]$ becomes $\left[(r+\delta) p_{K},(r+\delta) p_{K}\right]$ which is degenerate. Thus, the prescription to prevent the marginal revenue product of capital from leaving this degenerate interval is equivalent to adjusting the capital stock to maintain the marginal revenue product of capital always equal to $(r+\delta) p_{K}$.

Next consider irreversible investment. In this case, the sale price of capital, $b_{L}$, is zero and thus $R=\infty$. Therefore, $G=\infty$ and $G^{-1}=0$ so that $\Psi(G)=(r+\delta)\left(1-\gamma / \alpha_{P}\right)$ and $\Psi\left(G^{-1}\right)$ $=(r+\delta)\left(1-\gamma / \alpha_{N}\right)$. The interval $\left[\Psi(G) b_{L}, \Psi\left(G^{-1}\right) b_{U}\right]$ becomes $\left[0,(r+\delta)\left(1-\gamma / \alpha_{N}\right) b_{U}\right]$. In our model, no sales of capital are ever needed to prevent the marginal revenue product of capital from falling below the lower bound of this interval. However, the firm will sometimes need to purchase capital to prevent the marginal revenue product of capital from rising above the upper boundary of the interval. As emphasized in the irreversibility literature (see, for example, Pindyck $(1988,1991)$ and Dixit and Pindyck (1994)), the trigger value for the marginal revenue product in this case is higher than the standard Jorgensonian user cost $(r+\delta) b_{U}$. But as shown in the 
previous section, $(r+\delta) b_{U}$ is not the user cost of capital in this case. Instead, the user cost of capital is $\hat{c}_{U} \equiv\left(1-\gamma / \alpha_{N}\right)(r+\delta) b_{U}>(r+\delta) b_{U}$, and the optimal policy is to purchase capital to prevent the marginal revenue product of capital from rising above this user cost. ${ }^{15}$

Now consider irreversible disinvestment which is formally symmetric to the standard irreversible investment case. In this case, the sale price of capital, $b_{L}$, is positive and finite, and the purchase price of capital, $b_{U}$, is infinite, so that $R=\infty$. As in the case of irreversible investment, $G=\infty$ and $G^{-1}=0$ so that $\Psi(G)=(r+\delta)\left(1-\gamma / \alpha_{P}\right)$ and $\Psi\left(G^{-1}\right)=(r+\delta)\left(1-\gamma / \alpha_{N}\right)$. The interval $\quad\left[\Psi(G) b_{L}, \quad \Psi\left(G^{-1}\right) b_{U}\right] \quad$ becomes $\left[(r+\delta)\left(1-\gamma / \alpha_{p}\right) b_{L}, \infty\right]$. Notice that no purchases of capital are ever needed to prevent the marginal revenue product of capital from rising above the upper boundary of this interval. However, the firm will sometimes need to sell capital to prevent the marginal revenue product of capital from falling below the lower boundary of the interval, $\hat{c}_{L} \equiv\left(1-\gamma / \alpha_{P}\right)(r+\delta) b_{L}$, which is lower than the corresponding Jorgensonian user cost $(r+\delta) b_{L}$.

The consideration of irreversibility, in particular equations (30a) and (30c), leads to the following corollary.

Corollary to Proposition 3. For any finite $R \geq 1$, $\frac{G^{r}}{R}<\frac{1-\frac{\gamma}{\alpha_{N}}}{1-\frac{\gamma}{\alpha_{P}}}$.

${ }^{15}$ Dixit and Pindyck (1994, p. 145) thank Guiseppe Bertola for pointing out that the trigger value for the marginal revenue product of capital can be interpreted as the user cost of capital. However, neither they nor Bertola show that the value of the trigger is equal to the sum of the costs associated with interest, physical depreciation, and the expected capital loss on a unit of capital, as we have done in section IV. This demonstration gives economic content to an otherwise formal definition of user cost, and illustrates how this definition of user cost is based on Jorgenson's definition. 
Proof. Using Proposition 3, equations (30a,c) and the fact that $\Psi(x)$ is strictly decreasing we have $\frac{G^{\gamma}}{R}=\frac{\Psi\left(G^{-1}\right)}{\Psi(G)}<\frac{\Psi(0)}{\Psi(\infty)}=\frac{1-\frac{\gamma}{\alpha_{N}}}{1-\frac{\gamma}{\alpha_{p}}}$. q.e.d.

The importance of this corollary is that, along with Proposition 3, it defines a closed interval on the real line that contains $G \gamma_{/ R}$. Equivalently, given the capital price ratio $R$, Proposition 3 and its corollary define a closed interval on the real line that contains $G^{\gamma}$. Specifically, $G^{\gamma} \in\left[R, \frac{1-\gamma / \alpha_{N}}{1-\gamma / \alpha_{p}} R\right)$. According to Proposition 1, for $R \geq 1$ the optimal value of $G$ is the unique root of the equation $J(R, G)=0$ in this interval. This root can be calculated to any desired degree of precision in a finite number of steps using a simple algorithm: divide the interval in half, choose the half interval for which $J(R, G)$ is of opposite sign at the endpoints, and repeat this procedure until the desired degree of precision is achieved.

\section{A Local Approximation for the Optimal Value of $G$}

We have presented analytic results characterizing the optimal value of $G$ (see Propositions 1, 2, and 3 and the Corollary to Proposition 3), and we have described a finite algorithm to numerically compute the optimal value of $G$. In this section we present an approximate solution for the optimal value of $G$ that holds in the neighborhood of $R=1$. This approximation yields a simple expression that relates $G$ to the parameters of the problem facing the firm.

Recall that for a given value of $R$, the optimal value of $G$ satisfies $J(R, G)=0$. In Part D of the Appendix we show (equation (D.29)) that taking a Taylor's series approximation of $J(R, G)$ around the point $R=G=1$, and setting $J(R, G)$ equal to zero yields 


$$
\begin{aligned}
& -\frac{1}{6} J_{G G G}(1,1)(G-1)^{3} \cong \\
& \quad\left[J_{R}(1,1)+J_{R G}(1,1)(G-1)+\frac{1}{2} J_{R G G}(1,1)(G-1)^{2}\right](R-1)
\end{aligned}
$$

Equation (31) reflects the fact that $J_{G}(1,1)=0$ and $J_{G G}(1,1)=0$, so that the relationship between $R-1$ and $G-1$ is locally cubic at $R=G=1$. The terms involving $(G-1)$ and ( $G$ $1)^{2}$ in brackets on the right hand side of equation (31) are negligibly small in the neighborhood of $R=G=1$ and may be ignored. Part D of the Appendix (see equation (D.31)) shows that by substituting expressions for $J_{G G G}(1,1)$ and $J_{R}(1,1)$ into equation (31) we obtain

$$
G \cong 1+\left[\frac{6 \sigma_{y}^{2}}{n(r+\delta)}\right]^{1 / 3}(R-1)^{1 / 3} .
$$

According to equation (32) the wedge between the upper and lower boundary values of $y, G-1$, is proportional to the cube root of the wedge between the purchase and sale prices of capital, $R-1 .^{16}$ This cubic function displays the infinite value of $\mathrm{d} G / \mathrm{d} R$ at $R=1$ as presented in Proposition 2.

For a given value of $\gamma$, the wedge $G^{\gamma}$ between the upper and lower trigger values of the marginal revenue product of capital is an increasing function of $\sigma_{y}^{2}$. The intuitive explanation for this result is that an increase in the variance of $y$ shortens the expected length of time for $y$ to move from any given value to any other given value. Thus, if the boundaries $y_{L}$ and $y_{U}$ were to remain fixed, an increase in the variance of $y$ would shorten the expected length of time between changing the capital stock when $y$ is at one boundary, and then subsequently changing the capital stock in the opposite direction when $y$ is at the other boundary. The wedge between the purchase and sale prices of capital when $R>1$ means that

${ }^{16}$ In models in different economic contexts, Delgado and Dumas (1994) and Shreve and Soner (1994) have found a similar locally cubic property. 
it is costly to reverse a change in the capital stock, and by increasing the expected frequency of such reversals, an increase in the variance of $y$ would increase the expected costs facing the firm for given $y_{L}$ and $y_{U}$. To mitigate this increase in expected costs, the firm increases the wedge between the upper and lower boundaries.

For a given value of $\gamma$, the wedge $G^{\gamma}$ between the upper and lower trigger values of the marginal revenue product of capital is a decreasing function of $r+\delta$. An increase in $r$ means that the firm discounts the future more heavily and thus attaches less weight to the cost associated with future reversals of changes in the capital stock. Therefore, the firm is willing to incur a higher expected frequency of such reversals and narrows the wedge between the upper and lower boundaries of $y$. An increase in $\delta$ means that a smaller fraction of any current change in the capital stock will remain at any future date. Therefore, the expected cost associated with any future reversal of a current change in the capital stock will smaller. Thus, as in the case of an increase in $r$, the firm will choose to incur a higher expected frequency of such reversals and will narrow the wedge between the upper and lower trigger values.

Notice that the approximation for $G$ in equation (32) does not depend on $\mu_{y}$, the drift in $y$. The reason that $G$ is locally independent of the drift is that in the neighborhood of $R=1$, the optimal value of $G$ is very close to one, which means that the wedge between the upper and lower boundaries on $y$ is very small. The dynamics of $y$ from one boundary to the other boundary infinitesimally far away, and thus the expected costs associated with reversals of changes in the capital stock, are governed by $\sigma_{y} d z$ rather than by $\mu_{y} d t$ because $d t$ is of second order compared to $d z$. Thus $G$ is independent of $\mu_{y}$ in the neighborhood of $R=1$. 


\begin{tabular}{|l|l|c|}
\hline \multicolumn{3}{|c|}{ Table 1 } \\
\hline $\boldsymbol{R}$ & $\boldsymbol{G}$ & \\
\hline & exact & approximation \\
\hline 1.000001 & 1.021202 & 1.020977 \\
\hline 1.00001 & 1.046264 & 1.045194 \\
\hline 1.0001 & 1.102619 & 1.097367 \\
\hline 1.001 & 1.23737 & 1.209771 \\
\hline 1.01 & 1.623379 & 1.451938 \\
\hline 1.1 & 3.68156 & 1.973672 \\
\hline 1.5 & 22.2637 & 2.664956 \\
\hline 2 & 97.66962 & 3.097712 \\
\hline 3 & 749.9818 & 3.642952 \\
\hline \multicolumn{3}{|c|}{} \\
\hline$\mu_{X}=0.01, \sigma_{X}=0.2, \delta=0.1, r=0.03, \gamma=0.2$ \\
\hline
\end{tabular}

Table 1 illustrates the quality of the local approximation for an example in which $\mu_{X}=0.01, \sigma_{X}=0.2, \delta=0.1, r=$ 0.03 , and $\gamma=0.2$. Recognizing that $\sigma_{y}=\sigma_{X}=0.2$ and substituting the parameter values into equation (32), we have $G \cong 1+2(R-1)^{1 / 3}$. Table 1 illustrates that for tiny differences between the purchase and sale prices of capital, i.e., for values of $R$ very close to one, the approximation is very good. However, even for values of $R$ as small as 1.01 , the approximation shows signs of deteriorating in quality. When $R$ is 1.1 the exact value of $G$ differs from the approximation by almost a factor of two, and when $R=3$, the approximation differs from the exact value by more than a factor of 200 . Fortunately, as shown in Figure 1, the values of the upper and lower triggers $\left(c_{U}\right.$ and $\left.c_{L}\right)$ computed using these approximate values for $G$ can be very close to the values computed using the exact values of $G$. 


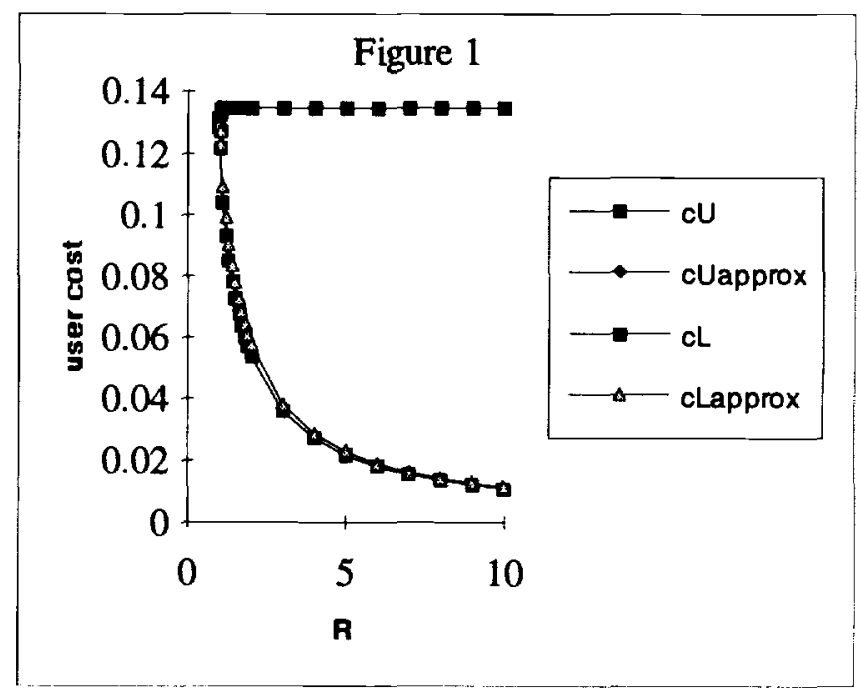

In Figure 1 , the value of the purchase price of capital $b_{U}$ is normalized to one, the value of the sale price of capital is $b_{L}=$ $1 / R$, and the other parameter values are the same as in Table 1. In this case, the user cost relevant for purchasing capital, $c_{U}$, equals $\Psi\left(G^{-1}\right)$, and the user cost relevant for selling capital, $c_{L}$, equals $\Psi(G) / R$. The fact that $R$ appears to have a much larger effect on the user cost $c_{L}$ than on the user cost $c_{U}$ in Figure 1 reflects our normalization in which $b_{U}$ is held fixed and $b_{L}$ falls as $R$ increases. As we have noted, the values of the user cost computed using the approximate values for $G$ are strikingly close to those computed using the exact values of $G$, perhaps surprisingly so in light of the poor quality of the approximation for values of $R$ greater than 1.1. (In fact, the exact and approximate values of $c_{U}$ are indistinguishable from each other in Figure 1.) These approximate values of the user cost turn out so close to the exact values because the user costs are most sensitive to the value of $G$ for values of $G$ close to one, and this is precisely the range of values where the approximation is quite good. For values of $R$ and $G$ outside the neighborhood of $R=G=1$, the functions $\Psi(G)$ and 
$\Psi\left(G^{-1}\right)$ are relatively insensitive to $G$, and thus the poor quality of the approximation for $G$ does not adversely affect the approximations of the user cost.

\section{The Quantitative Significance of Costly Reversibility}

The user costs of capital (which are the triggers for the marginal revenue product of capital) under costly reversibility are intermediate between the corresponding user costs in the cases of irreversibility and costless reversibility. To measure the extent to which the user costs under costly reversibility are closer to those under costless reversibility or under irreversibility, define the ratios $F_{L}(G)$ and $F_{U}(G)$ as

$$
\begin{aligned}
& F_{L}(G) \equiv \frac{c_{J}-c_{L}}{c_{J}-\hat{c}_{L}}=\frac{\Psi(1)-\Psi(G)}{\Psi(1)-\Psi(\infty)} \\
& F_{U}(G) \equiv \frac{c_{U}-c_{J}}{\hat{c}_{U}-c_{J}}=\frac{\Psi\left(G^{-1}\right)-\Psi(1)}{\Psi(0)-\Psi(1)},
\end{aligned}
$$

where a circumflex $\left({ }^{\wedge}\right)$ denotes the value of a user cost under complete irreversibility as in section V. Because $\Psi(x)$ is strictly decreasing in $x$, and $G \geq 1$, it follows that $0 \leq F_{i}(G) \leq 1$ and $F_{i}{ }^{\prime}(G)>0$ for $i=L, U$.

Treating the case of costless reversibility as the baseline case, the effect on the user costs relevant for purchasing capital is $\hat{c}_{U}-c_{J}>0$ under irreversibility and is $c_{U}-c_{J}>0$ under costly reversibility. Thus, the fraction $F_{U}(G)$ is the increase in the user cost due to costly reversibility relative to the increase due to irreversibility. Similarly, the fraction $F_{L}(G)$ is the decrease in the user cost relevant for selling capital due to costly reversibility relative to the decrease due to irreversibility. Because $F_{L}(G)$ and $F_{U}(G)$ are strictly increasing in $G$, which is strictly increasing in $R$, we see that an increase in $R$ causes the user costs to approach their values under irreversibility. 
Figure 2 presents the values of $F_{L}(G)$ and $F_{U}(G)$ for the numerical example introduced in section VI. The horizontal axis displays $1-1 / R$ which equals $\left(b_{U}-b_{L}\right) / b_{U}$, the wedge between the purchase and sale prices of capital expressed as a fraction of the purchase price. Notice that except for tiny values of the wedge, the user costs are much closer to those for irreversible investment than for reversible investment. For instance, with a mere $5 \%$ wedge between the purchase and sale prices of capital, the user costs are more than half of the distance toward the values that would arise under irreversibility $\left(F_{L}(G)=0.5815 ; F_{U}(G)=0.9839\right)$. Thus, even for a modest wedge between the purchase and sale prices, the values of the user costs are closer to the values computed under the assumption of complete irreversibility than to those computed under the assumption of costless reversibility.

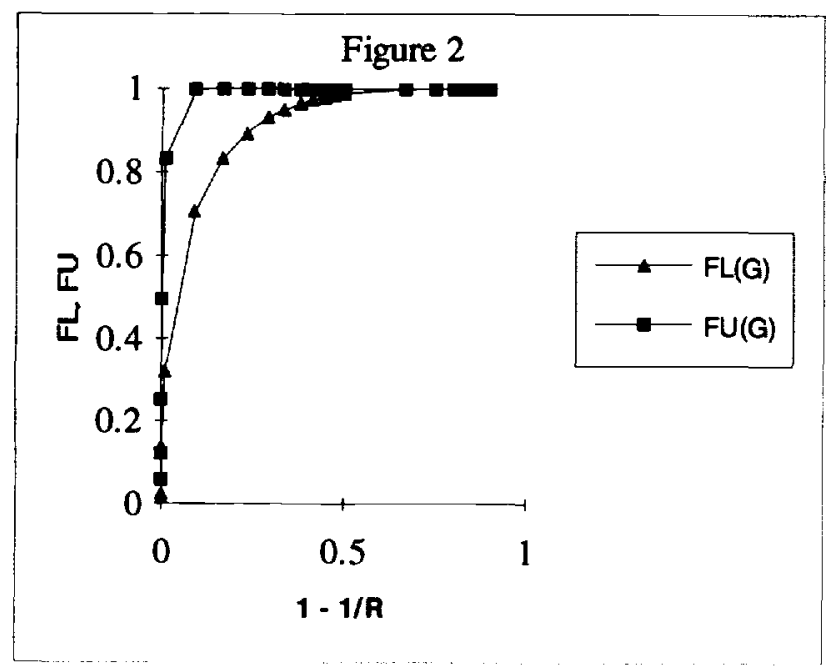




\section{Concluding Remarks}

Our point of departure was Arrow's observation that investment is generally reversible, but only at a cost. For the sake of simplicity, Arrow chose to examine the special case of irreversible investment, spawning a literature that has produced a variety of insights about optimal investment under uncertainty. A smaller literature has examined the general case of costly reversibility, with the result that optimal investment in this case is characterized by a two-trigger policy. Our paper contains three contributions to the understanding of optimal investment behavior in the presence of costly reversibility: (1) an explicit analytic solution to the optimal investment problem; (2) a finite algorithm to numerically compute this solution; and (3) a local approximation that provides a transparent view of the effects of various parameters on the values of the triggers.

In addition to solving the problem of costly reversibility in a parametric framework, our paper offers a prescription for optimal investment that encompasses the entire spectrum from costless reversibility to complete irreversibility. This prescription is based on a natural extension of Jorgenson's definition of the user cost of capital to the case of uncertainty. We define and calculate $c_{U}$, the user cost relevant for purchasing capital, and $c_{L}$, the user cost relevant for selling capital. Using these definitions, the prescription for optimal investment is simply stated: Purchase and sell capital as needed to prevent the marginal revenue product of capital from leaving the closed interval $\left[c_{L}, c_{U}\right]$. In the case of costless reversibility, the closed interval collapses to the single point $c_{L}=c_{U}$; maintaining the marginal revenue product of capital in this degenerate interval requires that the marginal revenue product of capital is continuously equated with the Jorgensonian user cost. In the case of irreversible investment, the lower bound of this interval is zero; for the cases discussed in the irreversibility literature (where the marginal revenue product of capital cannot possibly be negative), optimal investment can be completely characterized by only the upper bound of this interval, $c_{U}$. 
By casting the prescription for optimal investment in terms of the user cost of capital, our analysis takes a decidedly neoclassical perspective. In fact, the encompassing prescription for optimal investment makes the irreversible investment problem seem more neoclassical than it is presented in the irreversibility literature. For two reasons this neoclassical perspective is intended as a complement rather than a challenge to the major contributions of the irreversibility literature. First, the irreversibility literature provides a multitude of insights and techniques that facilitated the development and solution of the more encompassing model. Second, as a matter of economic substance, the considerations raised by the irreversibility literature appear to be quantitatively important. As we showed in section VII, the assumption of irreversibility provides a closer approximation to investment behavior than does the conventional assumption of costless reversibility, even when the transaction cost associated with the difference between the purchase and sale prices of capital is very small. 


\section{Appendix}

A. Identities involving roots of $\rho(\eta)=0$ in equation (12)

Applying the standard formula for the roots of a quadratic equation, we obtain

$$
\begin{gathered}
\alpha_{P}=\frac{-\left(\mu_{y}-\frac{1}{2} \sigma_{y}^{2}\right)+\sqrt{\left(\mu_{y}-\frac{1}{2} \sigma_{y}^{2}\right)^{2}+2 \sigma_{y}^{2}(r+\delta)}}{\sigma_{y}^{2}}>0 \\
\alpha_{N}=\frac{-\left(\mu_{y}-\frac{1}{2} \sigma_{y}^{2}\right)-\sqrt{\left(\mu_{y}-\frac{1}{2} \sigma_{y}^{2}\right)^{2}+2 \sigma_{y}^{2}(r+\delta)}}{\sigma_{y}^{2}}<0 \\
\alpha_{P} \alpha_{N}=-\frac{2(r+\delta)}{\sigma_{y}^{2}} \\
\rho(\eta)=-\frac{1}{2} \sigma_{y}^{2}\left(\eta-\alpha_{P}\right)\left(\eta-\alpha_{N}\right)
\end{gathered}
$$

Equation (A.3) implies

$$
\left(\gamma-\alpha_{P}\right)\left(\gamma-\alpha_{N}\right)=-\frac{2 \rho(\gamma)}{\sigma_{y}^{2}}
$$

Dividing equation (A.4) by equation (A.2) yields

$$
\frac{\left(\gamma-\alpha_{P}\right)\left(\gamma-\alpha_{N}\right)}{\alpha_{P} \alpha_{N}}=\frac{\rho(\gamma)}{r+\delta}
$$

\section{B. Properties of $\theta(x)$}

The function $\theta(x)$ is defined for $x>0$. This part of the Appendix shows that $\theta(x)$ is strictly increasing for $x>0$, and that $\theta^{\prime}(1), \theta^{\prime \prime}(1)$, and $\theta^{\prime \prime \prime}(1)$ are all finite. In addition, the values of $\theta(1)$ and $\theta^{\prime}(1)$ are computed. Observe that

$$
\theta(x) \equiv \frac{x^{\alpha_{P}}-x^{\gamma}}{x^{\alpha_{P}}-x^{\alpha_{N}}}=\frac{1-x^{\gamma-\alpha_{P}}}{1-x^{\alpha_{N}-\alpha_{P}}}
$$


Define $\quad b \equiv \alpha_{P}-\alpha_{N}>a \equiv \alpha_{P}-\gamma>0$ and rewrite equation (B.1) as

$$
\theta(x)=\frac{1-x^{-a}}{1-x^{-b}}
$$

It follows immediately from equation (B.2) that

$$
\theta(\infty)=1
$$

To calculate $\theta(0)$, multiply numerator and denominator of equation (B.2) by $x^{b}$ to obtain

$$
\theta(x)=\frac{x^{b}-x^{b-a}}{x^{b}-1}
$$

It follows immediately from equation (B.4) that

$$
\theta(0)=0
$$

To evaluate $\theta(x)$ and its derivatives at $x=1$, we will need to apply L'Hopital's Rule (repeatedly in the case of the derivatives).

Here we will evaluate $\theta(1)$ and $\theta^{\prime}(1)$ and we will show that $\theta^{\prime \prime}(1)$ and $\theta^{\prime \prime \prime}(1)$ are both finite. First we consider a function $f(x) \equiv \frac{n(x)}{d(x)}$ where $n(x)$ and $d(x)$ are infinitely differentiable. The derivative of this function with respect to $x$ is $f^{\prime}(x) \equiv \frac{N(x)}{D(x)}$ where $N(x) \equiv n^{\prime}(x) d(x)-n(x) d^{\prime}(x)$ and $D(x) \equiv[d(x)]^{2}$. Let $c$ be an arbitrary constant. If $N(c)=D(c)$ $=0$, then L'Hopital's Rule must be used to compute $f^{\prime}(c)$. Let $N^{(j)}(x)$ and $D^{(j)}(x)$ be the jth derivatives of $N(x)$ and $D(x)$ respectively, and let $J$ be the minimum value of $j$ for which $N^{(j)}(c)$ or $D^{(j)}(c)$ is not equal to zero. Provided that $N^{(J)}(c)$ is finite and $D^{(J)}(c)$ is not equal to zero, $f^{\prime}(c)$ is finite. Anticipating the need for higher order derivatives of $N(x)$ and $D(x)$ we calculate the first eight derivatives of each function, suppressing the argument $x$. 


$$
\begin{aligned}
N^{\prime}= & n^{\prime \prime} d-n d^{\prime \prime} \\
N^{\prime \prime}= & n^{\prime \prime \prime} d+n^{\prime \prime} d^{\prime}-n^{\prime} d^{\prime \prime}-n d^{\prime \prime \prime} \\
N^{\prime \prime \prime}= & n^{(4)} d+2 n^{\prime \prime \prime} d^{\prime}-2 n^{\prime} d^{\prime \prime \prime}-n d^{(4)} \\
N^{(4)}= & n^{(5)} d+3 n^{(4)} d^{\prime}+2 n^{\prime \prime \prime} d^{\prime \prime}-2 n^{\prime \prime} d^{\prime \prime \prime}-3 n^{\prime} d^{(4)}-n d^{(5)} \\
N^{(5)}= & n^{(6)} d+4 n^{(5)} d^{\prime}+5 n^{(4)} d^{\prime \prime}-5 n^{\prime \prime} d^{(4)}-4 n^{\prime} d^{(5)}-n d^{(6)} \\
N^{(6)}= & n^{(7)} d+5 n^{(6)} d^{\prime}+9 n^{(5)} d^{\prime \prime}+5 n^{(4)} d^{\prime \prime \prime}-5 n^{\prime \prime \prime} d^{(4)}-9 n^{\prime \prime} d^{(5)}-5 n^{\prime} d^{(6)}-n d^{(7)} \\
N^{(7)}= & n^{(8)} d+6 n^{(7)} d^{\prime}+14 n^{(6)} d^{\prime \prime}+14 n^{(5)} d^{\prime \prime \prime}-14 n^{\prime \prime \prime} d^{(5)}-14 n^{\prime \prime} d^{(6)}-6 n^{\prime} d^{(7)}-n d^{(8)} \\
N^{(8)}= & n^{(9)} d+7 n^{(8)} d^{\prime}+20 n^{(7)} d^{\prime \prime}+28 n^{(6)} d^{\prime \prime \prime}+14 n^{(5)} d^{(4)} \\
& -14 n^{(4)} d^{(5)}-28 n^{\prime \prime \prime} d^{(6)}-20 n^{\prime \prime} d^{(7)}-7 n^{\prime} d^{(8)}-n d^{(9)} \\
D^{\prime}= & 2 d d^{\prime} \\
D^{\prime \prime}= & 2 d^{\prime 2}+2 d d^{\prime \prime} \\
D^{\prime \prime \prime}= & 6 d^{\prime} d^{\prime \prime}+2 d d^{\prime \prime \prime} \\
D^{(4)}= & 6 d^{\prime \prime 2}+8 d^{\prime} d^{\prime \prime \prime}+2 d d^{(4)} \\
D^{(5)}= & 20 d^{\prime \prime} d^{\prime \prime \prime}+10 d^{\prime} d^{(4)}+2 d d^{(5)} \\
D^{(6)}= & 20 d^{\prime \prime \prime 2}+30 d^{\prime \prime \prime} d^{(4)}+12 d^{\prime} d^{(5)}+2 d d^{(6)} \\
D^{(7)}= & 70 d^{\prime \prime \prime} d^{(4)}+42 d^{\prime \prime \prime} d^{(5)}+14 d^{\prime} d^{(6)}+2 d d^{(7)} \\
D^{(8)}= & 70 d^{(4)^{2}}+112 d^{\prime \prime \prime} d^{(5)}+56 d^{\prime \prime} d^{(6)}+16 d^{\prime} d^{(7)}+2 d d^{(8)} \\
&
\end{aligned}
$$

Recalling the definition of $\theta(x)$ in equation (B.2) we have 
$\theta(x) \equiv \frac{n_{0}(x)}{d_{0}(x)}$ where $n_{0}(x) \equiv 1-x^{-a}$ and $d_{0}(x) \equiv 1-x^{-b}$ (B.6)

For any positive integer $\mathrm{j}$, the $\mathrm{j}$ th derivatives of $n_{0}(x)$ and $d_{0}(x)$ evaluated at $x=1$ are

$n_{0}^{(j)}(1)=(-1)^{j-1} \prod_{i=0}^{j-1}(a+i)$ and $d_{0}^{(j)}(1)=(-1)^{j-1} \prod_{i=0}^{j-1}(b+i)$.

Note that $n_{0}(1)=d_{0}(1)=0, n_{0}^{\prime}(1)=a$, and $d_{0}^{\prime}(1)=b$. Therefore, application of L'Hopital's Rule yields

$$
0<\theta(1)=\frac{a}{b}=\frac{\alpha_{P}-\gamma}{\alpha_{P}-\alpha_{N}}<1
$$

Define $N_{0}(x) \equiv n_{0}^{\prime}(x) d_{0}(x)-n_{0}(x) d_{0}^{\prime}(x)$ and

$D_{0}(x) \equiv\left[d_{0}(x)\right]^{2}$. Therefore, $N_{0}(1)=N_{0}^{\prime}(1)=0$, and

$N_{0}^{\prime \prime}(1)=n_{0}^{\prime \prime}(1) d_{0}^{\prime}(1)-n_{0}^{\prime}(1) d_{0}^{\prime \prime}(1)=a b(b-a)$; also,

$D_{0}(1)=D_{0}^{\prime}(1)=0$, and $D_{0}^{\prime \prime}(1)=2\left(d_{0}^{\prime}(1)\right)^{2}=2 b^{2}$.

\section{First derivative of $\theta(x)$.}

$$
\begin{gathered}
\text { Define } n_{1}(x) \equiv N_{0}(x)=n_{0}^{\prime}(x) d_{0}(x)-n_{0}(x) d_{0}^{\prime}(x) \text { and } \\
d_{1}(x) \equiv D_{0}(x)=\left[d_{0}(x)\right]^{2} \text {, and observe that } \theta^{\prime}(x) \equiv \frac{n_{1}(x)}{d_{1}(x)}
\end{gathered}
$$

Observe that $n_{1}(1)=n_{1}^{\prime}(1)=0, n_{1}^{\prime \prime}(1)=a b(b-a)$,

$d_{1}(1)=d_{1}^{\prime}(1)=0$, and $d_{1}^{\prime \prime}(1)=2 b^{2}$. Applying L'Hopital's

Rule, and using equation (A.4) we obtain

$$
\theta^{\prime}(1)=\frac{a(b-a)}{2 b}=\frac{\left(\alpha_{P}-\gamma\right)\left(\gamma-\alpha_{N}\right)}{2\left(\alpha_{P}-\alpha_{N}\right)}=\frac{\rho(\gamma)}{\sigma_{y}^{2}\left(\alpha_{P}-\alpha_{N}\right)}>0 \text {.(B.9) }
$$


Define $N_{1}(x) \equiv n_{1}^{\prime}(x) d_{1}(x)-n_{1}(x) d_{1}^{\prime}(x)$ and

$D_{1}(x) \equiv\left[d_{1}(x)\right]^{2}$. Note that $N_{1}^{(j)}(1)=D_{1}^{(j)}(1)=0, j=0,1,2,3$,

$N_{1}^{(4)}(1)=2\left(n_{1}^{(3)}(1) d_{1}^{\prime \prime}(1)-n_{1}^{\prime \prime}(1) d_{1}^{(3)}(1)\right)$, and

$D_{1}^{(4)}(1)=6 d_{1}^{\prime \prime 2}(1)=24 b^{4}$.

Second derivative of $\theta(x)$.

Define $n_{2}(x) \equiv N_{1}(x)$ and $d_{2}(x) \equiv D_{1}(x)$, and observe

that $\theta^{\prime \prime}(x) \equiv \frac{n_{2}(x)}{d_{2}(x)}$. Observe that

$n_{2}^{(j)}(1)=d_{2}^{(j)}(1)=0, j=0,1,2,3$,

$n_{2}^{(4)}(1)=2\left(n_{1}^{(3)}(1) d_{1}^{\prime \prime}(1)-n_{1}^{\prime \prime}(1) d_{1}^{(3)}(1)\right)$, and $d_{2}^{(4)}(1)=24 b^{4}$.

Therefore, $\theta^{\prime \prime}(1) \equiv \frac{n_{2}^{(4)}(1)}{d_{2}^{(4)}(1)}$ is finite. Define

$N_{2}(x) \equiv n_{2}^{\prime}(x) d_{2}(x)-n_{2}(x) d_{2}^{\prime}(x)$ and $D_{2}(x) \equiv\left[d_{2}(x)\right]^{2}$.

Note that $N_{2}^{(j)}(1)=D_{2}^{(j)}(1)=0, j=0,1, \ldots, 7$,

$N_{2}^{(8)}(1)=14\left(n_{2}^{(5)}(1) d_{2}^{(4)}(1)-n_{2}^{(5)}(1) d_{2}^{(4)}(1)\right)$, and

$D_{2}^{(8)}=70\left(d_{2}^{(4)}(1)\right)^{2}=70\left(D_{1}^{(4)}(1)\right)^{2}>0$.

Third derivative of $\theta(x)$.

Define $n_{3}(x) \equiv N_{2}(x)$ and $d_{3}(x) \equiv D_{2}(x)$, and observe

that $\theta^{\prime \prime \prime}(x) \equiv \frac{n_{3}(x)}{d_{3}(x)}$. Observe that

$n_{3}^{(j)}(1)=d_{3}^{(j)}(1)=0, j=0,1, \ldots, 7$,

$n_{3}^{(8)}(1)=14\left(n_{2}^{(5)}(1) d_{2}^{(4)}(1)-n_{2}^{(5)}(1) d_{2}^{(4)}(1)\right)$, and

$d_{3}^{(8)}(1)=70\left(D_{1}^{(4)}(1)\right)^{2}>0$. Therefore, $\theta^{\prime \prime \prime}(1) \equiv \frac{n_{3}^{(8)}(x)}{d_{3}^{(8)}(x)}$ is

finite.

We have already shown (equation (B.9)) that $\theta^{\prime}(1)>0$. To determine the sign of $\theta^{\prime}(x)$ for $x \neq 1$, note that $\theta^{\prime}(x)$ has 
the same sign as $N_{0}(x)$ because $D_{0}(x)>0$ for $x \neq 1$. Observe that $N_{0}(x)$ can be rewritten as

$$
N_{0}(x)=a b\left(\frac{x^{b}-1}{b}-\frac{x^{a}-1}{a}\right) x^{-(1+a+b)}
$$
lemma.

We can determine the sign of $N(x)$ using the following

Lemmo 1. Define $\varphi(z) \equiv \frac{x^{z}-\beta}{z}$ for $\beta \geq 1, x>0$, and $z>0$. If (a) $\beta>1$ or (b) $x \neq 1$, then $\varphi^{\prime}(z)>0$.

Proof. Observe that $\varphi(z)=\frac{e^{z \ln x}-\beta}{z}$ so that

$\varphi^{\prime}(z)=\frac{x^{2} \ln x}{z}-\frac{x^{2}-\beta}{z^{2}}=\frac{(z \ln x-1) x^{2}+\beta}{z^{2}}=\frac{\psi(z)}{z^{2}}$, where

$v(z) \equiv(z \ln x-1) x^{2}+\beta$.

Differentiating $v(z)$ yields

$v^{\prime}(z)=x^{z} \ln x+(z \ln x-1) x^{z} \ln x=z(\ln x)^{2} x^{z} \geq 0$ for $z>$

0 . Note that $k(0)=-1+\beta \geq 0$. Therefore, (a) if $\beta>1, k(z)>0$ for $z>0$ which implies that $\varphi^{\prime}(z)>0$ for $z>0$; (b) if $x \neq 1$,

$v^{\prime}(z)>0$ so $v(z)>0$ for $z>0$ which implies that $\varphi^{\prime}(z)>0$ for $z>0$. q.e.d.

Using the definition of $\varphi(z)$ in Lemma 1 above (setting the parameter $\beta$ equal to one), rewrite equation (B.10) as

$$
N_{0}(x)=a b(\varphi(b)-\varphi(a)) x^{-(1+a+b)}>0 \text { for } x \neq 1
$$

where the inequality follows from $b>a>0$ which implies $\varphi(b)>\varphi(a)$. Therefore, (since we have already shown that $\left.\theta^{\prime}(1)>0\right)$, we have

$$
\theta^{\prime}(x)>0
$$

\section{A Useful Lemma}

The following lemma will help in some subsequent derivations.

Lemma 2. $1-\frac{\gamma}{\alpha_{P}}+\theta(1)\left(\frac{-\gamma}{\alpha_{N}}+\frac{\gamma}{\alpha_{P}}\right)=\frac{\rho(\gamma)}{r+\delta}$. 
Proof. Note that

$$
1-\frac{\gamma}{\alpha_{P}}+\theta(1)\left(\frac{-\gamma}{\alpha_{N}}+\frac{\gamma}{\alpha_{P}}\right)=\frac{\alpha_{P}-\gamma}{\alpha_{P}}-\gamma \theta(1)\left(\frac{\alpha_{P}-\alpha_{N}}{\alpha_{P} \alpha_{N}}\right)
$$

Using equation (B.8) to substitute for $\theta(1)$ on the right hand side of equation (C.1) yields

$$
1-\frac{\gamma}{\alpha_{P}}+\theta(1)\left(\frac{-\gamma}{\alpha_{N}}+\frac{\gamma}{\alpha_{P}}\right)=\frac{\alpha_{P}-\gamma}{\alpha_{P}}-\eta\left(\frac{\alpha_{P}-\gamma}{\alpha_{P} \alpha_{N}}\right)
$$

Combining terms on the right hand side of equation (C.2) yields

$$
1-\frac{\gamma}{\alpha_{P}}+\theta(1)\left(\frac{-\gamma}{\alpha_{N}}+\frac{\gamma}{\alpha_{P}}\right)=\left(\frac{\alpha_{P}-\gamma}{\alpha_{P} \alpha_{N}}\right)\left(\alpha_{N}-\gamma\right)
$$

Using equation (A.5) to obtain an equivalent expression for the right hand side of equation (C.3) yields

$$
1-\frac{\gamma}{\alpha_{P}}+\theta(1)\left(\frac{-\gamma}{\alpha_{N}}+\frac{\gamma}{\alpha_{P}}\right)=\frac{\rho(\gamma)}{r+\delta}
$$

q.e.d.

\section{Properties of $J(R, G)$}

\section{Calculation of $J(R, 1)$ and $J(R, \infty)$}

Recall the definition of $J(R, G)$ in equation (17):

$$
J(R, G) \equiv R-G^{\gamma}-\frac{\gamma}{\alpha_{N}}\left(R-G^{\alpha_{N}}\right) \theta(G)-\frac{\gamma}{\alpha_{p}}\left(R-G^{\alpha_{r}}\right)(1-\theta(G))
$$

Setting $G=1$ in equation (D.1) yields

$$
J(R, 1)=(R-1)\left[1-\frac{\gamma}{\alpha_{N}} \theta(1)-\frac{\gamma}{\alpha_{P}}(1-\theta(1))\right]
$$

Using Lemma 2 to rewrite the right hand side of equation (D.2) yields 


$$
J(R, 1)=(R-1) \frac{\rho(\gamma)}{r+\delta}>0
$$

To evaluate $\lim _{G \rightarrow \infty} J(R, G)$, multiply equation (D.1) by $\left(G^{\alpha_{P}}-G^{\alpha_{N}}\right) /\left(G^{\alpha_{P}}-G^{\alpha_{N}}\right)$ and use the definition of $\theta(G)$ in equation (15) to obtain

$(R, G)=\frac{1}{G^{\alpha_{r}}-G^{\alpha_{\mu}}}\left[\left(R-G^{\gamma}\right)\left(G^{\alpha_{r}}-G^{\alpha_{n}}\right)-\frac{\gamma}{\alpha_{N}}\left(R-G^{a_{n}}\right)\left(G^{\alpha_{r}}-G^{y}\right)-\frac{\gamma}{\alpha_{p}}\left(R-G^{\alpha_{1}}\right)\left(G^{y}-G^{a_{\mu}}\right)\right]$

(D.4)

Now multiply equation (D.4) by $G^{-\alpha_{P}} / G^{-\alpha_{P}}$ to obtain

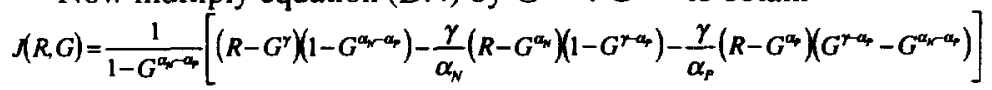

(D.5)

Next divide each term in brackets by $G^{\gamma}$ and multiply the expression in brackets by $G^{\gamma}$ to obtain

$\lambda(R, G)=\frac{G^{\gamma}}{1-G^{\alpha_{r}-\alpha_{p}}}\left[\left(R G^{-\gamma}-1\right)\left(1-G^{\alpha_{r-\alpha}}\right)-\frac{\gamma}{\alpha_{\mu}}\left(R G^{-r}-G^{\alpha_{r-\gamma} \gamma}\right)\left(1-G^{r-\alpha_{r}}\right)-\frac{\gamma}{\alpha_{p}}\left(R G^{-\gamma}-G^{\alpha_{r}-\gamma}\right)\left(G^{r-\alpha_{r}}-G^{\alpha_{r-\alpha}}\right)\right]$

Finally, let $G \rightarrow \infty$ and obtain

$\lim _{G \rightarrow-} J(R, G)=\left[-1+0+\frac{\gamma}{\alpha_{p}}\right] \lim _{G \rightarrow-} G^{\gamma}=-\left(\frac{\alpha_{P}-\gamma}{\alpha_{p}}\right) \lim _{G \rightarrow-} G^{\gamma}=-\infty$ (D.7)

\section{Calculation of Derivatives of $J(R, G)$}

Partially differentiating equation (D.1) with respect to $R$ $J_{R}(R, G)=1-\frac{\gamma}{\alpha_{N}} \theta(G)-\frac{\gamma}{\alpha_{P}}(1-\theta(G))=\frac{\alpha_{P}-\gamma}{\alpha_{P}}+\gamma \theta(G)\left(\frac{1}{\alpha_{P}}-\frac{1}{\alpha_{N}}\right)>0$ yields

Evaluating this derivative at $R=G=1$ and using Lemma 2

$$
J_{R}(1,1)=\frac{\rho(\gamma)}{r+\delta}
$$

Observe from equation (D.1) that $J(R, G)$ is linear in $R$ so that 


$$
J_{R R}(R, G) \equiv J_{R R R}(R, G) \equiv J_{R R G}(R, G) \equiv 0
$$

To compute $J_{G}(R, G)$, partially differentiate equation (D.1) with respect to $G$ to obtain

$J_{G}(R, G) \equiv-\gamma G^{\gamma-1}+\gamma G^{\alpha_{N-1}} \theta(G)-\frac{\gamma}{\alpha_{N}}\left(R-G^{\alpha_{N}}\right) \theta^{\prime}(G)+\gamma G^{\alpha_{p-1}}(1-\theta(G))+\frac{\gamma}{\alpha_{p}}\left(R-G^{\alpha_{\rho}}\right) \theta^{\prime}(G)$

(D.11)

Simplifying equation (D.11) yields

$J_{G}(R, G)=\frac{\gamma}{G}\left[-G^{\gamma}+G^{\alpha_{\rho}}+G(G)\left(G^{\alpha_{\mu}}-G^{\alpha_{\rho}}\right)\right]+\gamma \theta(G)\left[\frac{R-G^{\alpha_{\rho}}}{\alpha_{P}}-\frac{R-G^{\alpha_{\mu}}}{\alpha_{N}}\right]$

Observe that

$-G^{\gamma}+G^{\alpha_{P}}+\theta(G)\left(G^{\alpha_{N}}-G^{\alpha_{P}}\right)=-G^{\gamma}+G^{\alpha_{P}}-\left(G^{\alpha_{P}}-G^{\gamma}\right)=0$

so that

$$
J_{G}(R, G)=-\gamma \theta \cdot(G) M(R, G)
$$

where

$$
M(R, G) \equiv \frac{G^{\alpha_{P}}-R}{\alpha_{P}}-\frac{G^{\alpha_{N}}-R}{\alpha_{N}}
$$

Differentiating $M(R, G)$ with respect to $G$ we obtain $M_{G}(R, G) \equiv G^{a_{p}-1}-G^{\alpha_{N}-1}$ and $M_{G G}(R, G) \equiv\left(\alpha_{P}-1\right) G^{\alpha_{r}-2}-\left(\alpha_{N}-1\right) G^{\alpha_{N}-2}$. Therefore,

$$
\begin{aligned}
& M(1,1)=0 \\
& M_{G}(1,1)=0 \\
& M_{G G^{(1,1)}}=\alpha_{P}-\alpha_{N}
\end{aligned}
$$

It follows from equations (D.13) and (D.15a) and the fact that $\theta^{\prime}(1)$ is finite that

$$
J_{G}(1,1)=0
$$

Differentiating equation (D.13) with respect to $G$ yields

$$
J_{G G}(R, G)=-\gamma \theta^{\prime \prime}(G) M(R, G)-\gamma \theta^{\prime}(G) M_{G}(R, G) \quad(\mathrm{D} .17)
$$


Evaluating equation (D.17) at $R=G=1$, using equations (D.15a,b) and the fact that $\theta^{\prime}(1)$ and $\theta^{\prime \prime}(1)$ are both finite, we obtain

$$
J_{G G}(1,1)=0
$$

Differentiating equation (D.13) with respect to $R$ yields

$$
J_{G R}(R, G)=-\gamma \theta^{\prime}(G) M_{R}(R, G)
$$

Differentiating $M(R, G)$ in equation (D.14) with respect to $R$ yields

$$
M_{R}(R, G) \equiv-\left(\frac{1}{\alpha_{P}}-\frac{1}{\alpha_{N}}\right)
$$

Substituting equation (D.20) into equation (D.19) yields

$$
J_{G R}(R, G)=\gamma \theta \cdot(G)\left(\frac{1}{\alpha_{P}}-\frac{1}{\alpha_{N}}\right)
$$

Evaluating equation (D.21) at $R=G=1$ yields

$$
J_{G R}(1,1)=\gamma \theta \cdot(1)\left(\frac{1}{\alpha_{p}}-\frac{1}{\alpha_{N}}\right)
$$

Differentiating equation (D.21) with respect to $G$ and evaluating the result at $R=G=1$ yields

$$
J_{G G R}(1,1)=\gamma \theta "(1)\left(\frac{1}{\alpha_{P}}-\frac{1}{\alpha_{N}}\right)
$$

Differentiating equation (D.17) with respect to $G$ yields

$$
J_{G G G}(R, G)=-\chi\left(\theta^{\prime \prime \prime}(G) M(R, G)+2 \theta^{\prime \prime}(G) M_{G}(R, G)+\theta^{\prime}(G) M_{G G}(R, G)\right)
$$

(D.24)

Evaluate equation (D.24) at $R=G=1$ using equations (D.15a,b,c) and the fact that $\theta$ "(1) and $\theta^{\prime \prime \prime}(1)$ are finite to obtain

$$
J_{G G G}(1,1)=-\gamma \theta^{\prime}(1)\left(\alpha_{P}-\alpha_{N}\right) \quad \text { (D.25) }
$$

Using the expression for $\theta^{\prime}(1)$ in equation (B.9) we obtain

$$
J_{G G G}(1,1)=-\frac{w(\gamma)}{\sigma_{y}^{2}}
$$




\section{Approximation of $J(R, G)$ around $R=G=1$}

A third-order Taylor's series approximation of equation (D.1) around $R=G=1$ is

$J(R, G) \cong J(1,1)+J_{R}(1,1)(R-1)+J_{G}(1,1)(G-1)$

$+\frac{1}{2}\left[J_{R R}(1,1)(R-1)^{2}+2 J_{R G}(1,1)(R-1)(G-1)+J_{G G}(1,1)(G-1)^{2}\right]$

$+\frac{1}{6}\left[J_{R R A}(1,1)(R-1)^{3}+3 J_{\text {RRG }}(1,1)(R-1)^{2}(G-1)+3 J_{R G G}(1,1)(R-1)(G-1)^{2}+J_{G G G}(1,1)(G-1)^{3}\right]$

Observe that

$J(1,1)=J_{R R}(1,1) \equiv J_{R R R}(1,1) \equiv J_{R R G}(1,1) \equiv J_{G}(1,1) \equiv J_{G G}(1,1) \equiv 0$ so that

$$
\begin{aligned}
& J(R, G) \cong J_{R}(1,1)(R-1)+J_{R G}(1,1)(R-1)(G-1) \\
& +\frac{1}{6}\left[3 J_{R G G}(1,1)(R-1)(G-1)^{2}+J_{G G G}(1,1)(G-1)^{3}\right]
\end{aligned}
$$

Setting $J(R, G)=0$ in equation (D.28) yields

$$
-\frac{1}{6} J_{G G C}(1,1)(G-1)^{3} \equiv\left[J_{R}(1,1)+J_{R G}(1,1)(G-1)+\frac{1}{2} J_{R G G}(1,1)(G-1)^{2}\right](R-1)
$$

The terms involving $(G-1)$ and $(G-1)^{2}$ in brackets on the right hand side of equation (D.29) are negligibly small in the neighborhood of $R=G=1$, so that

$$
(G-1)^{3} \cong-6 \frac{J_{R}(1,1)}{J_{G G G}(1,1)}(R-1)
$$

Substituting equations (D.9) and (D.26) into equation (D.30) yields

$$
(G-1)^{3} \cong \frac{6 \sigma_{y}^{2}}{\gamma(r+\delta)}(R-1)
$$

\section{Proof that $J_{G}(R, G)<0$ when $J(R, G)=0$ and $R>1$} that

Observe from the definition of $J(R, G)$ in equation (D.1) 
$\frac{R-G^{\gamma}}{\gamma}-\frac{R-G^{\alpha_{P}}}{\alpha_{P}}=G(G)\left[\frac{R-G^{\alpha_{N}}}{\alpha_{N}}-\frac{R-G^{\alpha_{P}}}{\alpha_{P}}\right]$ when $J(R, G)=0$.

Rearranging equation (D.32), using the definition of $M(R, G)$ in equation (D.14), and assuming that $G \geq 1$ (to avoid $G=0$ since $Q(0)=0$ ) yields

$$
\left.M(R, G)\right|_{\mathcal{\prime}(R, G)=0}=\frac{1}{\theta(G)}\left[\frac{G^{\alpha_{p}}-R}{\alpha_{p}}-\frac{G^{\gamma}-R}{\gamma}\right]
$$

Observe from Lemma 1 that the term in brackets on the right hand side of equation (D.33) is positive for $R>1$. Also recall that $\theta(G)>0$ for $G>0$ so that equation (D.33) implies that

$$
\left.M(R, G)\right|_{(R, G)=0}>0 \quad \text { for } R>1
$$

Since $\theta^{\prime}(\mathrm{G})>0$ and $\gamma>0$ it follows from equations (D.34) and (D.13) that

$$
\left.J_{G}(R, G)\right|_{J(R, G)=0}<0 \text { for } R>1 .
$$

\section{E. Properties of $\Psi(x)$}

Recall the definition of $\Psi(x)$ in equation (18)

$$
\Psi(x) \equiv(r+\delta) \frac{\alpha_{p}-\gamma}{\alpha_{p}-\gamma \frac{(1-\theta(x))}{(1-\theta(1))}}
$$

Observe from equation (B.8) that

$1-\theta(1)=\left(\gamma-\alpha_{N}\right) /\left(\alpha_{P}-\alpha_{N}\right)$ so that equation (E.1) becomes

$$
\Psi(x)=(r+\delta) \frac{\alpha_{P}-\gamma}{\alpha_{P}-\gamma \frac{(1-\theta(x))}{\left(\gamma-\alpha_{N}\right)}\left(\alpha_{P}-\alpha_{N}\right)}
$$

Multiply the numerator and denominator of equation (E.2) by $\alpha_{N}-\gamma$ to obtain

$$
\Psi(x)=(r+\delta) \frac{\left(\alpha_{P}-\gamma\right)\left(\alpha_{N}-\gamma\right)}{\alpha_{P} \alpha_{N}-\alpha_{P} \gamma+\gamma(1-\theta(x))\left(\alpha_{P}-\alpha_{N}\right)}
$$

Dividing the numerator and denominator of equation (E.3) by $\alpha_{P} \alpha_{N}$ yields 


$$
\Psi(x)=(r+\delta) \frac{\left(\alpha_{P}-\gamma\right)\left(\alpha_{N}-\gamma\right) /\left(\alpha_{P} \alpha_{N}\right)}{1-\frac{\gamma}{\alpha_{N}}+\left(\frac{\gamma}{\alpha_{N}}-\frac{\gamma}{\alpha_{P}}\right)(1-\theta(x))}
$$

Using equation (A.5), we rewrite equation (E.4) as

$$
\Psi(x)=\frac{\rho(\gamma)}{1-\frac{\gamma}{\alpha_{N}} \theta(x)-\frac{\gamma}{\alpha_{p}}(1-\theta(x))}
$$

Recall from equation (14) that $\rho(\gamma)=h / H$ and use the definitions of $A$ and $B$ in equations $(20 \mathrm{a}, \mathrm{b})$ to obtain

$$
\Psi(G)=\frac{h}{H+A \theta(G)+B(1-\theta(G))}
$$

To derive an expression for $\Psi\left(G^{-1}\right)$ we use the following identities based on the definition of $\theta(x)$ in equation (15)

$$
G^{\alpha_{N}-\gamma} \theta(G)=\frac{G^{\alpha_{P}+\alpha_{N}-\gamma}-G^{\alpha_{N}}}{G^{\alpha_{P}}-G^{\alpha_{N}}} \frac{G^{-\alpha_{P}-\alpha_{N}}}{G^{-\alpha_{P}-\alpha_{N}}}=\frac{G^{-\gamma}-G^{-\alpha_{P}}}{G^{-\alpha_{N}}-G^{-\alpha_{p}}}=\theta\left(G^{-1}\right)
$$

and

$G^{\alpha_{r}-\gamma}(1-\theta(G))=\frac{G^{\alpha_{r}}-G^{\alpha_{\gamma^{+}+\alpha_{r}-\gamma}}}{G^{\alpha_{r}}-G^{\alpha_{N}}} \frac{G^{-\alpha_{r}-\alpha_{N}}}{G^{-\alpha_{r}-\alpha_{N}}}=\frac{G^{-\alpha_{N}}-G^{-\gamma}}{G^{-\alpha_{N}}-G^{-\alpha_{r}}}=1-\theta\left(G^{-1}\right)$

(E.8)

Observe from equation (E.6) that

$$
\Psi\left(G^{-1}\right)=\frac{h}{H+A \theta\left(G^{-1}\right)+B\left(1-G\left(G^{-1}\right)\right)}
$$

Substitute equations (E.7) and (E.8) into equation (E.9) and multiply the numerator and denominator by $G^{\gamma}$ to obtain

$$
\Psi\left(G^{-1}\right)=\frac{h G^{\gamma}}{H G^{\gamma}+A \theta(G) G^{\alpha_{N}}+B(1-G(G)) G^{\alpha_{r}}}
$$




\section{F. Verification of the Solution}

Verification of smooth-pasting condition, equation (10a)

Evaluate equation (21a) at the lower trigger $y_{L}$ to obtain

$$
q\left(y_{L}\right)=y_{L}^{\gamma}[H+A \theta(G)+B(1-\theta(G))]=\frac{h y_{L}^{\gamma}}{\Psi(G)}=b_{L}
$$

where the second equality follows from equation (E.6) and the third equality follows from equation (19a).

Verification of smooth-pasting condition, equation (10b)

Evaluate equation (21a) at the upper trigger $y_{U}$ to obtain

$$
q\left(y_{U}\right)=y_{L}^{\gamma} G^{\gamma}\left[H+A \theta(G) G^{\alpha_{N}-\gamma}+B(1-\theta(G)) G^{\alpha_{P}-\gamma}\right]
$$

Recall that $y_{U}^{\gamma}=y_{L}^{\gamma} G^{\gamma}$ and use equations (E.7) and (E.8) to rewrite equation (F.2) as

$$
q\left(y_{U}\right)=y_{U}^{r}\left[H+A \theta\left(G^{-1}\right)+B\left(1-\theta\left(G^{-1}\right)\right)\right]
$$

Finally, use equations (E.9) and (19b) to obtain

$$
q\left(y_{U}\right)=\frac{h y_{u}^{\gamma}}{\Psi\left(G^{-1}\right)}=b_{U}
$$

Verification of high-contact condition, equation (1la)

Differentiate equation (21a) with respect to $y$ to obtain

$q^{\prime}(y)=\gamma H y^{\gamma-1}+\alpha_{N} A \theta(G) y_{L}^{\gamma-\alpha_{N}} y^{\alpha_{N}-1}+\alpha_{P} B(1-\theta(G)) y_{L}^{\gamma-\alpha_{F}} y^{\alpha_{P}-1}$ (F.5)

Evaluate $q^{\prime}(y)$ at the lower trigger $y_{L}$ and use the definitions of $A$ and $B$ in equations (20a,b) to obtain

$$
q^{\prime}\left(y_{L}\right)=\gamma H y_{L}^{\gamma-1}[1-\theta(G)-(1-\theta(G))]=0
$$

\section{Verification of high-contact condition, equation (11b)}

Evaluate $q^{\prime}(y)$ in equation (F.5) at the upper trigger $y_{U}$ to obtain 
$q^{\prime}(y)=\gamma H y_{U}^{\gamma-1}+\alpha_{N} A \theta(G) y_{L}^{\gamma-\alpha_{N}} y_{U}^{\alpha_{N}-1}+\alpha_{p} B(1-\theta(G)) y_{L}^{\gamma-\alpha_{f}} y_{U}^{\alpha_{p}-1}$ (F.7)

Use the fact that $y_{U}^{\gamma}=y_{L}^{\gamma} G^{\gamma}$ and use the definitions of $A$ and $B$ in equations $(20 \mathrm{a}, \mathrm{b})$ to obtain

$$
q^{\prime}(y)=\gamma H y_{U}^{\gamma-1}\left[1-\theta(G) G^{\alpha_{N}-\gamma}-(1-\theta(G)) G^{\alpha_{p}-\gamma}\right]
$$

Finally, use equations (E.7) and (E.8) to rewrite equation (F.8) as

$$
q^{\prime}(y)=\gamma H y_{U}^{\gamma-1}\left[1-\theta\left(G^{-1}\right)-\left(1-\theta\left(G^{-1}\right)\right)\right]=0
$$

Verification that $J(R, G)=0$

Observe from the definition of $J(R, G)$ in equation (17) that

$$
H J(R, G)=R\left[H-\frac{\not H}{\alpha_{N}} \theta(G)-\frac{\not H}{\alpha_{p}}(1-\theta(G))\right]-G^{\gamma}\left[H-\frac{\not H}{\alpha_{N}} \theta(G) G^{\alpha_{N}-\gamma}-\frac{\not H}{\alpha_{p}}(1-\theta(G)) G^{\alpha_{r}-r}\right]
$$

Use the definitions of $A$ and $B$ in equations $(20 a, b)$ to obtain

$H J(R, G)=R[H+A \theta(G)+B(1-\theta(G))]-G^{\gamma}\left[H+A \theta(G) G^{\alpha_{x}-\gamma}+B(1-\theta(G)) G^{\alpha_{p}-\gamma}\right]$

(F.11)

Now use equations (E.6) - (E.9) to rewrite equation (F.11) as

$$
H J(R, G)=\frac{h R}{\Psi(G)}-\frac{h G^{\gamma}}{\Psi\left(G^{-1}\right)}
$$

Substitute equations (19a) and (19b) into equation (F.12) to obtain

$$
H J(R, G)=\frac{R b_{L}}{y_{L}^{\gamma}}-\frac{G^{\gamma} b_{v}}{y_{v}^{\gamma}}=\frac{b_{v}}{y_{L}^{\gamma}}-\frac{b_{v}}{y_{L}^{\gamma}}=0
$$

G. Proof of Proposition 1. We prove existence and uniqueness of the root of $J(R, G)=0$ separately for $R>1$ (Case I) and for $R=1$ (Case II).

Existence: Case I: Assume that $R>1$. Recall that $\rho(\gamma)>0$ and $r+\delta>0$. Therefore, it follows immediately from equation 
(D.3) that $J(R, 1)>0$. Recall from equation (D.7) that $\lim _{G \rightarrow \infty} J(R, G)=-\infty$. Therefore, since $J(R, G)$ is a continuous function of $G$ on the interval $[1, \infty)$, there is at least one root of $J(R, G)=0$ in $(1, \infty)$.

Case II: Assume $R=1$. Observe that $J(1,1)=0$ so that $G$ $=1$ is a root of the equation $J(1, G)=0$.

Uniqueness: Case I: Assume $R>1$. Recall from equation (D.35) that $\left.J_{G}(R, G)\right|_{J=0}<0$ when $R>1$. Therefore, since $J(R, G)$ is a continuous function of $G$ on the interval $[1, \infty)$, there is at most one root of $J(R, G)=0$ in this interval.

Case II: Assume $R=1$. To show that there are no roots other than $G=1$ in the interval $[1, \infty)$, it suffices to show that $J_{G}(1, G)<0$ for $G>1$. Recall that $\gamma \theta^{\prime}(G)>0$ so that equation (D.13) implies that $J_{G}(1, G)$ has the opposite sign as $M(1, G)=\frac{G^{\alpha_{P}}-1}{\alpha_{P}}-\frac{G^{\alpha_{N}}-1}{\alpha_{N}}$. Recall from equation (D.15a) that $M(1,1)=0$ and note that $M_{G}(1, G)=\left(G^{\alpha_{p}}-G^{\alpha_{N}}\right) G^{-1}>$ 0 for $G>1$. Thus $M(1, G)>0$ for $G>1$ and hence $J_{G}(1, G)<$ 0 for $G>1$.

Strictly increasing: Apply the implicit function to $J(R, G(R))=0$ to obtain

$$
G^{\prime}(R)=-\frac{\left.J_{R}(R, G)\right|_{J=0}}{\left.J_{G}(R, G)\right|_{J=0}} .
$$

Recall from equation (D.8) that $J_{R}(R, G)>0$, and recall from equation (D.35) that $J_{G}(R, G) J_{J=0}<0$ for $R>1$. Therefore, $G^{\prime}(R)>0$ for $R>1$. q.e.d.

\section{H. User Cost of Capital}

Recall that $y \equiv(X / K)$, so that $d y=\frac{d X}{K}-\frac{X}{K} \frac{d K}{K}=\mu_{y} y d t+\sigma_{y} y d z-y \frac{d K}{K}$, where $d \kappa$ is the increment to accumulated gross investment. Using Ito's Lemma and the fact that $q(y)$ in equation (21) is twice continuously differentiable yields 
$d q(y)=\left[\mu_{y} y q^{\prime}(y)+\frac{1}{2} \sigma_{y}^{2} y^{2} q^{\prime \prime}(y)\right] d t+\sigma_{y} q^{\prime}(y) d z-\frac{d K}{K} y q^{\prime}(y)$.

(H.1)

At the boundaries of the range of inaction, where gross investment is nonzero, the high-contact conditions in equations (11a) and (11b) must hold; these require that $q^{\prime}\left(y_{L}\right)=q^{\prime}\left(y_{H}\right)=0$. Since $d x$ is finite even when it is nonzero, the product $d \kappa y q^{\prime}(y) / K$ evaluated at the boundaries is zero. ${ }^{17}$ Since all terms in equation (H.1) involving $q^{\prime}(y)$ are zero at $y=y_{L}$ and $y=y_{U}$,

$$
E\left\{d q \mid y=y_{i}\right\} / d t=\frac{1}{2} \sigma_{y}^{2} y_{i} q^{\prime \prime}\left(y_{i}\right) \text { for } i=L, U
$$

\section{Disinvestment at the lower boundary}

Define the user cost of capital relevant for disinvestment as

$$
c_{L} \equiv\left(r+\delta-\frac{E\left\{d q \mid y=y_{L}\right\} / d t}{q\left(y_{L}\right)}\right) b_{L}
$$

Differentiating equation (21a) twice with respect to $y$, and evaluating the second derivative at $y=y_{L}$ yields

$y_{L}^{2} q^{\prime \prime}\left(y_{L}\right)=H y_{L}^{\gamma} \gamma(\gamma-1)+A \theta(G) y_{L}^{\gamma} \alpha_{N}\left(\alpha_{N}-1\right)+B(1-\theta(G)) y_{L}^{\gamma} \alpha_{P}\left(\alpha_{P}-1\right)$

Use the definitions of $H, A$, and $B$ in equations (14), (20a), and (20b) respectively to obtain

$y_{L}^{2} q^{\prime \prime}\left(y_{L}\right)=\frac{h}{\rho(\gamma)} y_{L}^{\gamma}\left[\gamma(\gamma-1)-\gamma A \theta(G)\left(\alpha_{N}-1\right)-\gamma(1-\theta(G))\left(\alpha_{P}-1\right)\right]$

Use equation (A.4) to obtain an expression for $\sigma_{y}^{2}$ and substitute this expression and equation (H.S) into equation (H.2) to obtain

${ }^{17}$ Control at the boundaries is infinitesimal, so the capital stock does not "jump". Also note that $1 / K$ is finite if the bounds are finite. 
$E\left\{d q \mid y=y_{L}\right\} / d t=\frac{-h}{\left(\gamma-\alpha_{p}\right)\left(\gamma-\alpha_{N}\right)} y_{Y}^{Y}\left[\gamma(\gamma-1)-\gamma \theta(G)\left(\alpha_{N}-1\right)-\gamma(1-\theta(G))\left(\alpha_{P}-1\right)\right]$

Recall from equation (F.1) that

$$
q\left(y_{L}\right)=\frac{h y_{L}^{\gamma}}{\Psi(G)}
$$

Dividing equation (H.6) by equation (H.7) yields

$\frac{E\left\{d q \mid y=y_{L}\right\} / d t}{q\left(y_{L}\right)}=-\frac{\gamma(\gamma-1)-\gamma \theta(G)\left(\alpha_{N}-1\right)-\gamma(1-\theta(G))\left(\alpha_{P}-1\right)}{\left(\gamma-\alpha_{P}\right)\left(\gamma-\alpha_{N}\right)} \Psi(G)$

Observe from equation (E.4) that

$(r+\delta)=\frac{\alpha_{P} \alpha_{N}}{\left(\alpha_{P}-\gamma\right)\left(\alpha_{N}-\gamma\right)}\left[1-\frac{\gamma}{\alpha_{N}}+\left(\frac{\gamma}{\alpha_{N}}-\frac{\gamma}{\alpha_{P}}\right)(1-\theta(G))\right] \Psi(G)$

Now subtract equation (H.8) from equation (H.9) to obtain $(r+\delta)-\frac{E\left\{d q \mid y=y_{L}\right\} \gamma d t}{q\left(y_{L}\right)}=\frac{1}{\left(\alpha_{p}-\gamma\right)\left(\alpha_{N}-\gamma\right)}\left[\begin{array}{l}\alpha_{p} \alpha_{N}-\gamma \alpha_{p}+\gamma\left(\alpha_{p}-\alpha_{N}\right)(1-\theta(G))+\gamma^{2}-\gamma \\ -\gamma \alpha_{N}+\gamma+\gamma\left(\alpha_{N}-1\right)(1-\theta(G))-\gamma\left(\alpha_{p}-1\right)(1-\theta(G))\end{array}\right] \Psi(G)$

(H.10)

Simplifying equation (H.10) yields

$$
(r+\delta)-\frac{E\left\{d q \mid y=y_{L}\right\} / d t}{q\left(y_{L}\right)}=\frac{\alpha_{P} \alpha_{N}-\gamma \alpha_{P}+\gamma^{2}-\gamma \alpha_{N}}{\left(\alpha_{P}-\gamma\right)\left(\alpha_{N}-\gamma\right)} \Psi(G)=\Psi(G)
$$

Finally, substituting equation (H.11) into equation (H.3) yields

$$
c_{L}=\Psi(G) b_{L}
$$


Investment at the upper boundary

Define the user cost of capital relevant for (positive) investment as

$$
c_{v} \equiv\left(r+\delta-\frac{E\left\{d q \mid y=y_{u}\right\} / d t}{q\left(y_{v}\right)}\right) b_{v}
$$

Differentiating equation (21b) twice with respect to $y$, and evaluating the second derivative at $y=y_{U}$ yields

$y_{U} q^{\prime \prime}\left(y_{U}\right)=H y_{U}^{\gamma} \gamma(\gamma-1)+A \theta\left(G^{-1}\right) y_{U}^{\gamma} \alpha_{N}\left(\alpha_{N}-1\right)+B\left(1-\theta\left(G^{-1}\right)\right)_{y_{U}^{\gamma}}^{\gamma} \alpha_{P}\left(\alpha_{P}-1\right)$

(H.14)

Use the definitions of $H, A$, and $B$ in equations (14), (20a), and $(20 \mathrm{~b})$ respectively to obtain

$y_{U} q^{\prime \prime}\left(y_{U}\right)=\frac{h}{\rho(\gamma)} y_{U} \gamma\left[\gamma(\gamma-1)-\gamma \theta\left(G^{-1}\right)\left(\alpha_{N}-1\right)-\gamma\left(1-\theta\left(G^{-1}\right)\right)\left(\alpha_{P}-1\right)\right]$

(H.15)

Use equation (A.4) to obtain an expression for $\sigma_{y}^{2}$ and substitute this expression and equation (H.15) into equation (H.2) to obtain

$E\left\{d q \mid y=y_{\nu}\right\} / d t=\frac{-h}{\left(\gamma-\alpha_{P}\right)\left(\gamma-\alpha_{N}\right)} y_{\nu}{ }^{r}\left[\gamma(\gamma-1)-\gamma \theta\left(G^{-1}\right)\left(\alpha_{N}-1\right)-\gamma\left(1-\theta\left(G^{-1}\right)\right)\left(\alpha_{P}-1\right)\right]$ (H.16)

Recall from equation (F.4) that

$$
q\left(y_{v}\right)=\frac{h y_{u}^{r}}{\Psi\left(G^{-1}\right)}
$$

Dividing equation (H.16) by equation (H.17) yields

$$
\frac{E\left\{d q \mid y=y_{U}\right\} / d t}{q\left(y_{U}\right)}=-\frac{\gamma(\gamma-1)-\gamma \theta\left(G^{-1}\right)\left(\alpha_{N}-1\right)-\gamma\left(1-\theta\left(G^{-1}\right)\right)\left(\alpha_{\mathrm{P}}-1\right)}{\left(\gamma-\alpha_{P}\right)\left(\gamma-\alpha_{N}\right)} \Psi\left(G^{-1}\right)
$$


Observe from equation (E.4) that

$(r+\delta)=\frac{\alpha_{P} \alpha_{N}}{\left(\alpha_{P}-\gamma\right)\left(\alpha_{N}-\gamma\right)}\left[1-\frac{\gamma}{\alpha_{N}}+\left(\frac{\gamma}{\alpha_{N}}-\frac{\gamma}{\alpha_{P}}\right)\left(1-\theta\left(G^{-1}\right)\right)\right] \Psi\left(G^{-1}\right)$

(H.19)

Now subtract equation (H.18) from equation (H.19) to obtain

$(r+\delta)-\frac{E\left\{d q \mid y=y_{v}\right\} / d t}{q\left(y_{U}\right)}=\frac{1}{\left(\alpha_{p}-\gamma\right)\left(\alpha_{N}-\gamma\right)}\left[\begin{array}{l}\alpha_{r} \alpha_{N}-\gamma \alpha_{r}+\gamma\left(\alpha_{r}-\alpha_{N}\right)\left(1-\theta\left(G^{-1}\right)\right)+\gamma^{2}-\gamma \\ -\gamma \alpha_{N}+\gamma+\gamma\left(\alpha_{N}-1\right)\left(1-\theta\left(G^{-1}\right)\right)-\gamma\left(\alpha_{r}-1\right)\left(1-\sigma\left(G^{-1}\right)\right)\end{array}\right] \Psi\left(G^{-1}\right)$

(H.20)

Simplifying equation (H.20) yields

$(r+\delta)-\frac{E\left\{d q \mid y=y_{U}\right\} / d t}{q\left(y_{U}\right)}=\frac{\alpha_{P} \alpha_{N}-\gamma \alpha_{P}+\gamma^{2}-\gamma \alpha_{N}}{\left(\alpha_{P}-\gamma\right)\left(\alpha_{N}-\gamma\right)} \Psi\left(G^{-1}\right)=\Psi\left(G^{-1}\right)$

(H.21)

Finally, substituting equation (H.21) into equation (H.13) yields

$$
c_{v}=\Psi\left(G^{-1}\right) b_{v}
$$




\section{References}

Arrow, Kenneth J., "Optimal Capital Policy with Irreversible Investment" in Value, Capital and Growth, Papers in honour of Sir John Hicks, J.N. Wolfe (ed.), Chicago: Aldine Publishing Company, 1968, pp. 1-19.

Bertola, Guiseppe, "Adjustment Costs and Dynamic Factor Demands: Investment and Employment Under Uncertainty," Ph.D. Dissertation, Cambridge, MA: Massachusetts Institute of Technology, 1988.

Bertola, Guiseppe and Ricardo Caballero, "Irreversibility and Aggregate Investment," Review of Economic Studies, 61 (1994), 223-246.

Delgado, Francisco and Bernard Dumas, "How Far Apart Can Two Riskless Interest Rates Be? (One Moves, the Other One Does Not), Rodney L. White Center for Financial Research, The Wharton School of the University of Pennsylvania, Working Paper No. 18-94, August 1994.

Dixit, Avinash, "Entry and Exit Decisions under Uncertainty," Journal of Political Economy, 97, 3 (June 1989), 620-638.

Dixit, Avinash and Robert S. Pindyck, Investment Under Uncertainty, Princeton, NJ: Princeton University Press, 1994.

Dumas, Bernard, "Super Contact and Related Optimality Conditions," Journal of Economic Dynamics and Control, 15, 4 (October 1991), 675-685.

Jorgenson, Dale W., "Capital Theory and Investment Behavior," American Economic Review Papers and Proceedings, 53 (May 1963), 247-259.

Pindyck, Robert S., "Irreversible Investment, Capacity Choice, and the Value of the Firm," American Economic Review, 78, 5 (December 1988), 969-985. 
Pindyck, Robert S., "Investment, Uncertainty, and Investment," Journal of Economic Literature, 29 (September 1991), 1110-1148.

Shreve, S.E. and H.M. Soner, "Optimal Investment and Consumption with Transaction Costs," Annals of Applied Probability, 4, 3 (1994), 609-692. 